\title{
Developmental Roles of the Hog1 Protein Phosphatases of the Maize Pathogen Cochliobolus heterostrophus
}

\author{
Rina Zuchman ${ }^{1,2}{ }^{\mathbb{D}}$, Roni Koren ${ }^{1}$ and Benjamin A. Horwitz ${ }^{1, *(\mathbb{D})}$ \\ 1 Faculty of Biology, Technion-Israel Institute of Technology, Haifa 3200003, Israel; rinaz@technion.ac.il (R.Z.); \\ ronik@technion.ac.il (R.K.) \\ 2 Smoler Protein Center, Technion-Israel Institute of Technology, Haifa 3200003, Israel \\ * Correspondence: horwitz@technion.ac.il; Tel.: +972-48-293-976
}

check for

updates

Citation: Zuchman, R.; Koren, R.; Horwitz, B.A. Developmental Roles of the Hog1 Protein Phosphatases of the Maize Pathogen Cochliobolus

heterostrophus. J. Fungi 2021, 7, 83. https://doi.org/10.3390/jof7020083

Academic Editor: Brian L. Wickes

Received: 1 October 2020

Accepted: 19 January 2021

Published: 26 January 2021

Publisher's Note: MDPI stays neutral with regard to jurisdictional claims in published maps and institutional affiliations.

Copyright: (C) 2021 by the authors. Licensee MDPI, Basel, Switzerland. This article is an open access article distributed under the terms and conditions of the Creative Commons Attribution (CC BY) license (https:// creativecommons.org/licenses/by/ $4.0 /)$.

\begin{abstract}
Protein phosphorylation cascades are universal in cell signaling. While kinome diversity allows specific phosphorylation events, relatively few phosphatases dephosphorylate key signaling proteins. Fungal mitogen activated protein kinases (MAPK), in contrast to their mammalian counterparts, often show detectable basal phosphorylation levels. Dephosphorylation, therefore, could act as a signal. In Cochliobolus heterostrophus, the Dothideomycete causing Southern corn leaf blight, ferulic acid (FA) — an abundant phenolic found in plant host cell walls-acts as a signal to rapidly dephosphorylate the stress-activated MAP kinase Hog1 (High Osmolarity Glycerol 1). In order to identify the protein phosphatases responsible, we constructed mutants in Hog1 phosphatases predicted from the genome by homology to yeast and other species. We found that Cochliobolus heterostrophus mutants lacking PtcB, a member of the PP2C family, exhibited altered growth, sporulation, and attenuated dephosphorylation in response to FA. The loss of the dual-specificity phosphatase CDC14 led to slow growth, decreased virulence, and attenuated dephosphorylation. Mutants in two predicted tyrosine phosphatase genes PTP1 and PTP2 showed normal development and virulence. Our results suggest that a network of phosphatases modulate Hog1's dual phosphorylation levels. The mutants we constructed in this work provide a starting point to further unravel the signaling hierarchy by which exposure to FA leads to stress responses in the pathogen.
\end{abstract}

Keywords: protein phosphatases; plant pathogen; plant phenolics; ferulic acid; plant defense; signaling pathways; mitogen activated protein kinase (MAPK); stress response

\section{Introduction}

Protein phosphorylation cascades are universal in cell signaling. Signaling protein activity depends on a balance between protein phosphorylation and dephosphorylation. The vast array of kinases allows for a high degree of specificity in cellular phosphorylation events. In contrast, a relatively small number of phosphatases are responsible for the dephosphorylation of key signaling proteins. For example, the genome of the opportunistic fungal pathogen Aspergillus fumigatus encodes 110 kinases (Kinomer databasehttp:/ / www.compbio.dundee.ac.uk/kinomer/bin/kinomes.pl searched September 2020), while 32 phosphatase catalytic subunits have been identified [1]. In most eukaryotes, kinases comprise as much as $2 \%$ of the genome, and are responsible for the phosphorylation of more than $30 \%$ of the cellular proteins [2]. The specificity of phosphorylation is derived mainly from the high diversity of the kinome. Phosphatase activity is important for the avoidance of the over-activation of biological processes, and the spatial and temporal restriction of kinase activation [3]. The specificity of phosphatase activity depends, among other properties of the enzyme and substrates, on the amino acid to be dephosphorylated (e.g., Ser/Thr or Tyr; S/T or Y) and on the additional proteins mediating the phosphorylation/dephosphorylation reactions [4,5].

The early work on the response of yeast to hyperosmotic stress characterized the high osmolarity/stress activated MAPK (Mitogen Activated Protein Kinase) Hog1 (High 
Osmolarity Glycerol 1). Upon the exposure of the cell to high osmolarity, Hog1 undergoes hyperphosphorylation, promoting its translocation to the nucleus. In the nucleus, Hog1 stimulates the expression of a set of genes involved in glycerol production and uptake, and thus balances the osmotic differences and prevents cell death [6]. Most fungal genomes encode 3-4 MAP kinases, which are central to development and virulence of pathogenic species [7]. Hog1 orthologs of filamentous fungi play roles not only in stress tolerance but also in development and virulence.

The exposure of the maize pathogen Cochliobolus heterostrophus to ferulic acid (FA) results in the rapid dephosphorylation of Hog1 [8]. FA, a ubiquitous plant phenolic present in the cell walls of stems and leaves, is thought to act as an antimicrobial defense molecule. It is covalently linked to lignin and proteins by ether bonds, and to polysaccharides by ester bonds, acting as a universal connector between cell wall polymers. Upon a plant-pathogen encounter, FA is released by fungal esterases. FA plays an important role in strengthening the cell wall by anchoring lignin to the wall polysaccharides, thus restricting the accessibility of plant pathogens $[9,10]$. The exposure of the fungus to FA causes hyphal shrinkage, membrane damage, and regulated cell death $[8,11,12]$. Previous work revealed that FA acts as a stressor, causing rapid cell death with some hallmarks of programmed/regulated cell death (PCD/RCD) [7,12]. Quite unexpectedly, exposure to FA strongly suppressed dual phosphorylation at the TXY motif of the MAPKs Chk1/Pmk1 and $\operatorname{Hog} 1[8]$.

Usually, dual threonine and tyrosine phosphorylation (at the TXY motif) activates MAPKs in response to signals. We propose two hypotheses to explain why FA exposure dephosphorylates Hog1, rather than raising the dual-phosphorylation level. (1) Lowering Hog1 phosphorylation mitigates a cell-death-promoting signal. In support of this, data from a yeast link sustained Hog1 phosphorylation to cell death $[13,14]$. Likewise, the fungicide fludioxonil causes the sustained activation of Hog1, killing fungal cells [15]. Furthermore, the deletion of Hog1 increased the sensitivity of $C$. heterostrophus to FA toxicity [8]. (2) The dephosphorylation of Hog1 could, in itself, transduce a host-derived signal provided by FA. In support of hypothesis (2), exposure to FA extensively reprograms the fungal transcriptome [16]. These two hypotheses are not mutually exclusive. Both the lack of active Hog1 and its over-activation could compromise survival. If so, the homeostasis of the Hog1 dual-phosphorylation levels would be essential for tolerance toward FA. In order to investigate these two mechanisms and the role of dephosphorylation, we identified phosphatase genes.

The genome of $C$. heterostrophus, the agent of Southern corn leaf blight, was among the first Dothideomycetes sequenced [11,17]. In fungi, several phosphatases have been proposed as Hog1 modulators: PtcB, a S/T phosphatase of the PP2C family [1]; CDC14, a cell-divisionrelated phosphatase with dual specificity; and PTP1 and PTP2, tyrosine phosphatases [18-20]. PtcB was identified as a high osmolarity glycerol response phosphatase in Aspergillus fumigatus; a $\triangle$ PtcB strain showed increased SakA (Hog1 ortholog) and MpkA phosphorylation, the higher expression of osmostress-dependent genes, higher sensitivity to cell-wall-damaging agents, and impaired biofilm formation [1]. PtcB orthologs regulate Hog1's phosphorylation state in vivo, both at the basal level and during adaptation to stress, via dephosphorylation at Thr-171, which is located in the activation loop along with Tyr-173 [19,21].

CDC14 is studied in the context of cell division, but its role in multi-stress responses has generally been overlooked, although it could interact with other phosphatases and MAPKs [22,23]. There is evidence that CDC14 and Hog1 are somehow linked; for instance, Hog1 phosphorylation is reduced in Beauveria bassiana mutants lacking CDC14 [24], and a theoretical study modelling the cell cycle effects of osmotic stress in yeast predicted that the two are indirectly connected [25]. Despite these hints, Hog1 was not recovered among the physical interactors of CDC14 in a proteomic study on Candida albicans [26], suggesting that further work is required in order to clarify the nature of the interaction or link between these two proteins. 
PTP2 overexpression in Magnaporthe oryzae counteracted the action of fludioxonil [15]. This suggests that PTP2 orthologs might serve as endogenous regulators of Hog1. Indeed, in Cryptococcus neoformans, Ptp2 suppressed Hog1 hyperphosphorylation, and its activity was required for wild-type vegetative growth, sexual differentiation, stress responses, antifungal drug resistance, and virulence factor regulation. Ptp2 apparently acts in C. neoformans through the negative-feedback loop of the HOG pathway. Interestingly, the absence of PTP2 did not compromise its viability, since PTP1 served as a complementary PTP to control some of the stress responses [27]. Collectively, the findings from the diverse fungal species detailed above suggest that the four candidate genes-PtcB, CDC14, Ptp1 and Ptp2-could serve as Hog1 modulators in $\mathrm{C}$. heterostrophus. Here, we set out to examine this possibility by exploring the effects of FA on the Hog1 pathway, characterizing, by gene deletion experiments, the role of the aforementioned genes in the dephosphorylation of Hog1.

\section{Materials and Methods}

\subsection{Candidate Protein Phosphatase Gene Search}

Amino acid sequences of AfPtcB (A. fumigatus), ScCDC14 (Saccharomyces cerevisiae), ScPTP1, and MoPTP2 (M. oryzae) protein phosphatase genes $[15,18,19]$ were retrieved from the Uniprot Database (http://www.Uniprot.org/). Reciprocal BLASTP searches of the JGI C. heterostrophus $\mathrm{C} 4(\mathrm{Ch})$ database led to the predicted orthologs in C. heterostrophus. Phylogenetic trees were constructed with the Phylogeny.fr pipeline-MUSCLE (alignment), GBLOCKS (gap curation), PhyML (phylogeny) and TreeDyn (tree plotting) at http:/ / www.phylogeny.fr/ [28]. The phylogenetic trees included the predicted C. heterostrophus candidate orthologous genes from alignments with different fungi such as $A$. fumigatus $(A f)$, S. cerevisiae (Sc), M. oryzae (Mo), C. neoformans (Cf), Alternaria alternata (Aa) and other organisms representing outgroups.

\subsection{DNA Manipulations and Construction of C. heterostrophus Transformation Vectors}

Split marker PCR products ( 10-15 g total) were designed (primer sequences are given in Table 1), in order to replace the genes of interest (see Table 2: PtcB, CDC14, PTP1 and PTP2) with a hygromycin resistance cassette as described by Catlett at al. [29]. DNA fragments were amplified and cloned using the pGEM ${ }^{\circledR}$-T Easy Vector Systems (Promega, Madison, WI, USA) according to the manufacturer's instructions. Plasmids were transformed into E. coli DH5 $\alpha$ and extracted using A Mini-Prep DNA extraction kit (Macherey-Nagel, Düren, Germany). The plasmids were linearized using restriction enzymes, and introduced into the fungi by protoplast transformation (as detailed below).

Table 1. Primer sequences for the construction of $C$. heterostrophus transformation vectors and the verification of mutants. Hph, hygromycin resistance cassette, see Methods.

\begin{tabular}{|c|c|c|c|c|}
\hline Gene & JGI Protein ID & Synonym & Sequence $\left(5^{\prime}\right.$ to $\left.3^{\prime}\right)$ & Origin \\
\hline \multirow[t]{4}{*}{ Hygromycin } & & M13Rhyg & AGCGGATAACAATTTCACACAGGA & $\begin{array}{c}\text { pUCATPH seq. 2865-2888RC- }-5^{\prime} \text { region } \\
\text { of hygB }\end{array}$ \\
\hline & & NLC37 & GGATGCCTCCGCTCGAAGTA & 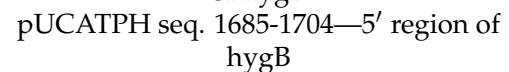 \\
\hline & & M13Fhyg & CGCCAGGGTTTTCCCAGTCACGAC & $\begin{array}{c}\text { pUCATPH seq. } 352-375-3^{\prime} \text { region } \\
\text { of hygB }\end{array}$ \\
\hline & & NLC38 & CGTTGCAAGACCTGCCTGAA & $\begin{array}{c}\text { pUCATPH seq. } \begin{array}{c}2132-2150 R C-3^{\prime} \text { region } \\
\text { of hygB }\end{array}\end{array}$ \\
\hline \multirow[t]{8}{*}{ PtcB } & 200919 & FP1 & GGGAGCATGTAGTAAGTGC & forward primer to PtcB $5^{\prime}$ flank \\
\hline & & RP1 & $\begin{array}{c}\text { TCCTGTGTGAAATTG TTATC- } \\
\text { CGCTCTTGTCCGGTATCTAGGAGGAC }\end{array}$ & $\begin{array}{l}\text { complementary to M13R-reverse } \\
\text { primer to PtcB } 5^{\prime} \text { flank }\end{array}$ \\
\hline & & & GTCGTGACTGGGAAAACCCTGG & complementary to M13F-forward \\
\hline & & $\mathrm{FP2}$ & CGCTTGTGCTCACTTGACCTGG & primer to PtcB $3^{\prime}$ flank \\
\hline & & RP2 & GAATCAGTGGCCAGATCCAGTC & reverse primer to PtcB $3^{\prime}$ flank \\
\hline & & ORF FR & GGCCAGACACTCTCCGAGC & \\
\hline & & ORF RV & СTTCTCGCTTGTCTTCTTGGC & \\
\hline & & HI-Upstream & CTCAAGTAGAGGTGAGTATGGG & \\
\hline
\end{tabular}


Table 1. Cont.

\begin{tabular}{|c|c|c|c|c|}
\hline Gene & JGI Protein ID & Synonym & Sequence $\left(5^{\prime}\right.$ to $\left.3^{\prime}\right)$ & Origin \\
\hline \multirow[t]{8}{*}{ CDC14 } & \multirow[t]{8}{*}{56964} & FP1 & GGTGCATGTTCAGGGACGGCC & \multirow{8}{*}{$\begin{array}{l}\text { forward primer to CDC14 } 5^{\prime} \text { flank } \\
\text { complementary to M13R-reverse } \\
\text { primer to CDC14 } 5^{\prime} \text { flank } \\
\text { complementary to M13F-forward } \\
\text { primer to CDC14 } 3^{\prime} \text { flank } \\
\text { reverse primer to CDC14 } 3^{\prime} \text { flank }\end{array}$} \\
\hline & & RP1 & $\begin{array}{l}\text { TCCTGTGTGAAATTGTTA TCCGCT- } \\
\text { GAGCAGGAAGGGGCGACTCTC }\end{array}$ & \\
\hline & & & GTCGTGACTGGGAAAACCCTGGCGC & \\
\hline & & FP2 & CACTTCTTAGTCTCCATCTCCATCGC & \\
\hline & & RP2 & ATCGGCAAAGACCCTGCCCGTC & \\
\hline & & ORF FR & CGACTTCCTTGCCTTTGCC & \\
\hline & & ORF RV & GTTCTCGTCAATGCGCTGG & \\
\hline & & HI-Upstream & GTGGGGGGAGTTTAGTTGC & \\
\hline \multirow[t]{8}{*}{ PTP1 } & \multirow[t]{8}{*}{142461} & FP1 & GATGCGATACGATGGTAGGG & \multirow{8}{*}{$\begin{array}{c}\text { forward primer to PTP1 } 5^{\prime} \text { flank } \\
\text { complementary to M13R-reverse } \\
\text { primer to PTP1 } 5^{\prime} \text { flank } \\
\text { complementary to M13F-forward } \\
\text { primer to PTP1 } 3^{\prime} \text { flank } \\
\text { reverse primer to PTP1 } 3^{\prime} \text { flank }\end{array}$} \\
\hline & & RP1 & $\begin{array}{l}\text { TCCTGTGTGAAATTGTTATCCGCTCG } \\
\text { CCCTTGAGCTTTGCCTTG }\end{array}$ & \\
\hline & & & GTCGTGACTGGGAAAACCCTGG & \\
\hline & & FP2 & CGCTTGGGCGTGAGAACAATGC & \\
\hline & & $\mathrm{RP} 2$ & CCAGACGACAACCGCTAATGAAC & \\
\hline & & ORF FR & CTGCTAGAGACGTCAATGCC & \\
\hline & & ORF RV & GCACAATCCGCGGGTTGGTAG & \\
\hline & & HI-Upstream & CAAAGGGGACATGGACGCAC & \\
\hline \multirow[t]{9}{*}{ PTP2 } & \multirow[t]{9}{*}{169617} & FP1 & GGCTTTAGCTTGCGATGGTC & \multirow{9}{*}{$\begin{array}{c}\text { forward primer to PTP2 } 5^{\prime} \text { flank } \\
\text { complementary to M13R-reverse } \\
\text { primer to PTP2 } 5^{\prime} \text { flank } \\
\text { complementary to M13F-forward } \\
\text { primer to PTP2 } 3^{\prime} \text { flank } \\
\text { reverse primer to PTP2 } 3^{\prime} \text { flank }\end{array}$} \\
\hline & & & TCCTGTGTGAAATTGTTAT & \\
\hline & & KP1 & CCGCTCGAGACGTGAACGTGGGAAG & \\
\hline & & FP2 & GTCGTGACTGGGAAAACCCTGGC & \\
\hline & & 112 & GGGCAAAGCTCATTGGGAG & \\
\hline & & $\mathrm{RP} 2$ & CAAGGAAACACAATGCCCCG & \\
\hline & & ORF FR & CCAACAAACCCAATGCACTCGTC & \\
\hline & & ORF RV & GTAGGGCCATCCGTGCTAG & \\
\hline & & HI-Upstream & CGCCTTTGCTGTCGTCGCC & \\
\hline
\end{tabular}

Table 2. Candidate protein phosphatase gene search.

\begin{tabular}{|c|c|c|c|c|c|c|}
\hline Family $^{a}$ & Subfamily ${ }^{b}$ & Class/Domain $^{c}$ & Ch Model Name ${ }^{d}$ & JGI Protein ID $^{\mathrm{e}}$ & $\begin{array}{c}\text { N. crassa } \\
\text { Homolog Gene }\end{array}$ & NCU $\mathrm{g}$ \\
\hline $\mathrm{S} / \mathrm{T}$ & PPM & PP2Cc & estExt_Genewise1Plus.C_19_t10368 & 200919 & pph-8 & 04600 \\
\hline PTP & Dual-specificity & DSPc & fgenesh1_pm.3_\#_471 & 56964 & cdc-14 & 03246 \\
\hline PTP1 & Classical & PTPc & e_gw1.16.187.1 & 142461 & pty-2 & 02257 \\
\hline PTP2 & Classical & PTPc & estExt_Genewise1.C_12_t30017 & 169617 & pty-3 & 05364 \\
\hline
\end{tabular}

${ }^{a}$ Family abbreviations: S/T, serine/threonine; PTP, protein tyrosine phosphatase. ${ }^{b}$ Subfamily abbreviations: PPM, Mg2+ or Mn2+dependent protein phosphatase; dual-specificity, serine/threonine and tyrosine phosphatase. ${ }^{c}$ Class/domain abbreviations: PP2Cc, protein phosphatase 2C catalytic subunit; DSPc, dual-specificity phosphatase catalytic subunit; PTPc, protein tyrosine phosphatase catalytic subunit. d,e From the JGI C. heterostrophus genome project (https://mycocosm.jgi.doe.gov/pages/search-for-genes.jsf?organism=CocheC4_1). $\mathrm{f}, \mathrm{g}$ Based on gene tables from Ghosh et al. [18], reciprocal BLASTP searches of the JGI C. heterostrophus C4 database, and the construction of phylogenetic trees using the Phylogeny.fr pipeline (MUSCLE (alignment), GBLOCKS (gap curation), PhyML (phylogeny), and TreeDyn (tree plotting) at http:/ /www.phylogeny.fr/ [28].

\subsection{Generation and Transformation of Fungal Protoplasts.}

Fungal protoplasts were generated and transformed as described in Turgeon et al. [30]. In brief, C. heterostrophus, race T strain C4, was grown in $90 \mathrm{~mm}$ Petri dishes with (complete medium, xylose, $\mathrm{CMX}$ ), at $22^{\circ} \mathrm{C}$ for about 1 week, in a $12 \mathrm{~h}$ light $/ 12 \mathrm{~h}$ dark regime. A conidial suspension from these plates was germinated overnight, as follows: the spores were inoculated to $150 \mathrm{~mL}$ (complete medium, CM), in a rotary shaker for $18 \mathrm{~h}$ at $30^{\circ} \mathrm{C}, 200 \mathrm{rpm}$, and used for the preparation of the protoplasts. The fungal suspension was centrifuged at $8000 \mathrm{rpm}$ for 10 min (Sorvall, Thermo Fisher Scientific, Waltham, MA, USA; SLA1500 rotor). The resulting mycelium sediment was suspended with $70 \mathrm{~mL}$ enzyme-osmoticum solution (see [30]; $\beta$ glucanase was replaced by an equivalent amount, following calibration, of VinoTaste enzyme preparation, Novozymes A/S, Bagsvaerd, Denmark) and divided into five $125 \mathrm{~mL}$ Erlenmeyer flasks, followed by incubation for $2.5 \mathrm{~h}$ at $30^{\circ} \mathrm{C}, 70 \mathrm{rpm}$, in a rotary shaker. The protoplasts released were filtered through gauze. The filtrate was then centrifuged at $4500 \mathrm{rpm}$ for $5 \mathrm{~min}$ at $4{ }^{\circ} \mathrm{C}$. The protoplast pellets were washed with $10 \mathrm{~mL} 0.7 \mathrm{M} \mathrm{NaCl}$, followed by a second wash in $10 \mathrm{~mL}$ sorbitol-Tris-calcium (reagents were from Sigma, Saint Louis, MO, USA, unless noted otherwise) buffer (STC, see [30]). 
The resulting protoplasts were suspended in $200 \mu \mathrm{L}$ of STC and counted. A concentration of $10^{8}$ cells $/ \mathrm{mL}$ was obtained, and of these, $10^{7}$ protoplasts were used for the transformation. The protoplasts were incubated on ice with $25 \mu \mathrm{g}$ DNA for $12 \mathrm{~min}$ (a negative control without DNA was subjected to the same procedure). Polyethylene glycol was added in three aliquots of 200,200, and $800 \mu \mathrm{L}$ each, and then diluted with $1 \mathrm{~mL}$ STC and plated for regeneration. A 1\% agar overlay containing $100 \mathrm{\mu g} / \mathrm{mL}$ Hygromycin B (HYG, catalog number H0654, Sigma, Saint Louis, MO, USA) was added after 18 h, for selection. For the protoplast viability determination, one of the control plates was overlayed with medium without HYG.

\subsection{Single-Spore Isolation}

Newly-formed colonies after transformation were transferred to CMX-HYG $100 \mu \mathrm{g} / \mathrm{mL}$ plates. Once formed, the spores were collected and diluted in sterile water so that up to 10 spores were dispersed on a $25 \mathrm{~mL}$ CMX-HYG $100 \mu \mathrm{g} / \mathrm{mL}$ plate, resulting in the formation of new colonies, each originating from a single spore.

\subsection{Transformant Verification}

The gene replacement by the Hygromycin resistance cassette (HYG) was verified by PCR. Amplification with primers designed from the gene sequence (ORF FR and ORF RV, Table 1) confirmed the loss of the ORF, to levels below detection. Amplification with a primers upstream of the $5^{\prime}$ gene flank used to construct the knockout mutant (HI Upstream, Table 1) paired with a primer within the hygromycin phosphotransferase cassette (NLC37 and NLC38, Table 1) confirmed the replacement of the desired gene by the HYG resistance marker.

\subsection{Growth Rate Measurements and Phenotypes}

C. heterostrophus strains were grown in CM agar plates with or without $1 \mathrm{M}$ sorbitol, and on complete medium with dimethylsulfoxide (CM-DMSO) agar plates with or without $2 \mathrm{mM}$ FA (DMSO is the solvent for FA stock; the final DMSO concentration $0.15 \% v / v$ ), for up to 14 days $(8 \mathrm{~h} / 16 \mathrm{~h}$ dark/light cycles). The radial hyphal growth was measured every 1-2 days, and the colony morphology was imaged using an Olympus (Olympus Life Science, Waltham, MA, USA) SZX16 fluorescence binocular microscope. The spores were imaged using a Leica (Leica Camera AG, Wetzlar, Germany) DMI8 inverted microscope, and the colonies were imaged using a cellphone camera. After 14 days, conidia from an area of $4 \mathrm{~cm}^{2}$ were collected in $0.8 \mathrm{~mL}$ liquid $\mathrm{CM}$ and filtered through double layered gauze in order to clear residual hyphae from the spore suspension. In total, $5 \mu \mathrm{L}$ were allowed to set on a cover slip in a humid chamber for $1.5 \mathrm{~h}$. Pictures were taken using a Leica DMI8 inverted microscope. For the adhesion assays, each droplet was washed twice with $50 \mu \mathrm{L}$ pure deionized water (DDW) and documented again. The image processing was performed using an LAS X program (https:/ / www.leica-microsystems.com/products/ microscope-software/p/leica-las-x-ls/).

\subsection{Hog1's Phosphorylation State in Response to Different Stimuli}

C. heterostrophus strains ( 8 cubes of about $8 \mathrm{~mm}^{3}$, taken from the margins of wild type (WT), dPTP1 and dPTP2, and 20 cubes of $\mathrm{dPtcB}$ and dCDC14 colonies) were each grown in $200 \mathrm{~mL} \mathrm{CM}$ in a shake culture $\left(24^{\circ} \mathrm{C}, 185 \mathrm{rpm}\right)$ for $40 \mathrm{~h}$. Each was then divided equally into four Erlenmeyer flasks, followed by the addition of either sorbitol or FA (1 M and $2 \mathrm{mM}$ final concentrations, respectively). For the sorbitol and FA, liquid CM and CM supplemented with the solvent, and DMSO at matching concentration $(0.15 \% v / v)$ served as controls, respectively. The fungus was further incubated for 5 and $30 \mathrm{~min}$, respectively, and then separated from the liquid medium by filtration on gauze, and immediately frozen in liquid nitrogen. 


\subsection{Immunoblotting}

Frozen mycelia from C. heterostrophus strains were ground in liquid nitrogen, and their proteins were extracted using SDS buffer containing 5\% SDS, $100 \mathrm{mM}$ Tris $\mathrm{pH}$ 8, and $10 \mathrm{mM}$ DTT, heated for $5 \mathrm{~min}$ at $95^{\circ} \mathrm{C}$ and sonicated ( $30 \mathrm{~s}$, level 5, Microson XL-Misonic, Farmingdale, NY, USA). The extract was then centrifuged (10,000 g, $10 \mathrm{~min}$, room temperature), and the supernatant was subjected to acetone precipitation. The resolved protein pellet was solubilized using a urea buffer containing $8 \mathrm{M}$ urea, $100 \mathrm{mM}$ ammonium bicarbonate and $10 \mathrm{mM}$ DTT. The protein concentration was determined using the Bradford assay, and $25 \mu \mathrm{g}$ from each extract was loaded onto a 10\% SDS-PAGE. The transfer was performed using the e-blot protein transfer kit (GenScript, Piscataway, NJ, USA). The blots were exposed to either p-P38 antibody (Cell Signaling, Danvers, MA, USA, T180-Y182, 9211S) or Hog1 antibody (Santa Cruz Biotechnology, Dallas, TX, USA, y-215, sc-9079) in order to examine the changes in the Hog1 phosphorylation levels or the total Hog1 levels, respectively, upon exposure to different treatments (sorbitol or FA). A luminescence detection by ECL was performed in a Fusion Pulse (VILBER Lourmat, Collégien, France) imager.

\subsection{Virulence Assays}

Seedlings of sweet corn (local hybrid, Royalty) were grown for about 3 weeks, until the fourth leaf began to expand. Next, $1 \mathrm{~mm}^{3}$ cubes of $C$. heterostrophus strains were attached to the leaf using clear tape in a moist environment (a closed plastic bag) and allowed to set for $24 \mathrm{~h}$, after which the tape was removed and the plants were grown for four more days. The lesion areas were measured using ImageJ (public software, National Institutes of Health, Bethesda, MD, USA).

\subsection{Complementation Analysis}

In order to rule out the possibility that the phenotypes detected in the PtcB deletion mutants resulted from other mutations, a complementation analysis (AddBack [AB]) was performed. The PtcB genomic sequence was amplified from C. heterostrophus C4 using a PCR reaction performed with FP1 and RP2 (Table 1). The complementation was performed by co-transformation, with the full PtcB gene and plasmid pNG conferring neomycin resistance. The colonies growing through the overlay with $1 \mathrm{mg} / \mathrm{mL}$ neomycin underwent single sporing, as described above. The transformants were verified by PCR, first by amplification with primers designed from the gene sequence (ORF) verifying the insertion of the gene, and subsequently with primers from the hygromycin phosphotransferase cassette, in order to determine whether the insertion of the gene replaced the hygromycin resistance cassette at the PtcB locus. The phenotypes were tested for three AB transformants that were positive for the ORF.

\section{Results}

\subsection{Identification of Protein Phosphatase Genes}

The four putative Hog1 modulators presented in the Introduction section, each containing a different structural phosphatase superfamily domain, were searched with BLASTP. Predicted orthologs were identified (Table 2, Figure 1 and Figure S1). C. heterostrophus knock-out mutants were then generated in order to examine the effects of the deletion of each of these genes. Four independent transformants of dPtcB, three of dCDC14, three of dPTP1, and four of dPTP2 were generated. The gene deletion by homologous integration was verified by PCR (Figure 2; see Methods section for details). In each case, the desired gene was indeed missing (negative for ORF). The Hygromycin (HYG) resistance cassette, which was inserted as a substitution for the existing gene, and as a selection marker, was indeed inserted in the correct location (positive for homologous integration) (Figure 2). The insertion of a wild type (WT) copy of PtcB restored the normal phenotypes. In these add-back $(\mathrm{AB})$ strains, the insertions were ectopic, as the hygromycin resistance cassette was retained following single-conidia isolation; the transformants acquired neomycin resistance as well as the complete PtcB ORF (Figures S2 and S3). Perhaps due to the severe 
phenotypes, we could not obtain transformants in the complementation experiments for $\mathrm{dCDC} 14$. The indistinguishable phenotypes of three independently-isolated deletion transformant lines, however, rule out, as far as possible, the possibility that the phenotypes we observed resulted from other mutations.

A

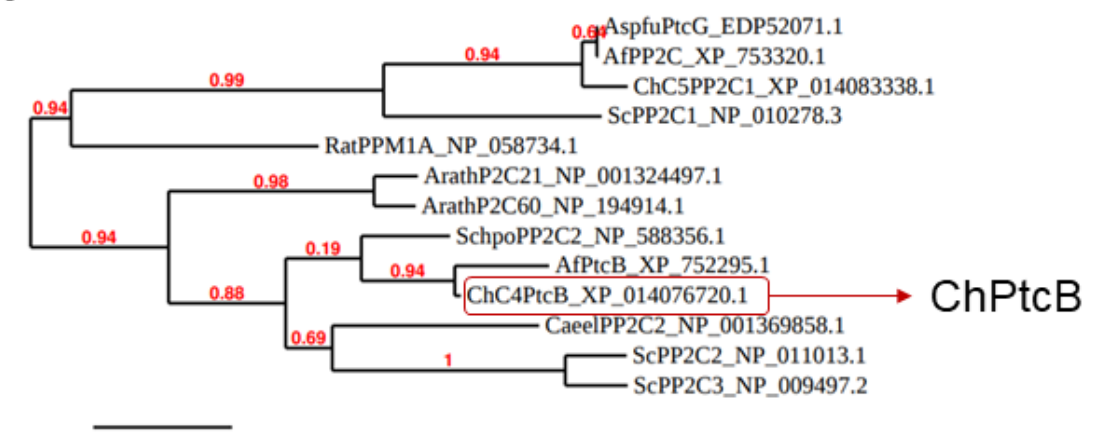

0.2

B

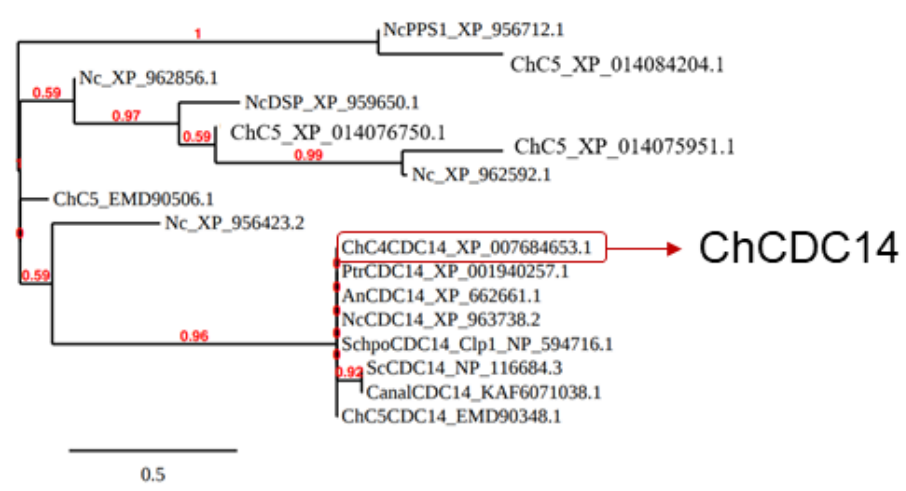

C

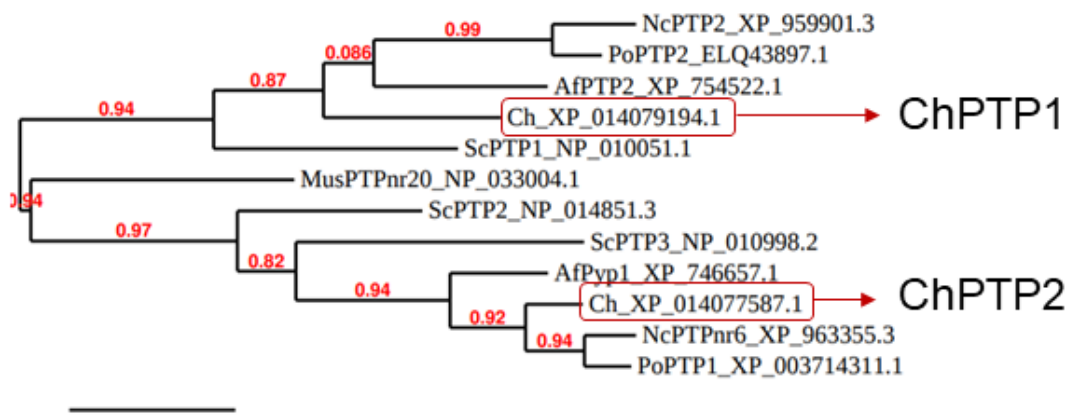

0.3

Figure 1. C. heterostrophus candidate phosphatase-encoding genes. (A-C) Following reciprocal BLASTP searches, the phylogenetic trees of the predicted C. heterostrophus phosphatase candidate ortholog genes were generated using the Phylogeny.fr pipeline (for details, see Methods and the legend to Table 2). Each entry in the trees starts with an informative gene name (organism: Nc, Neurospora crassa; Ch, Cochliobolus heterostrophus; Mus, mouse, Cael, C. elegans) followed by an abbreviated phosphatase class name and, last, the NCBI protein database ID. 


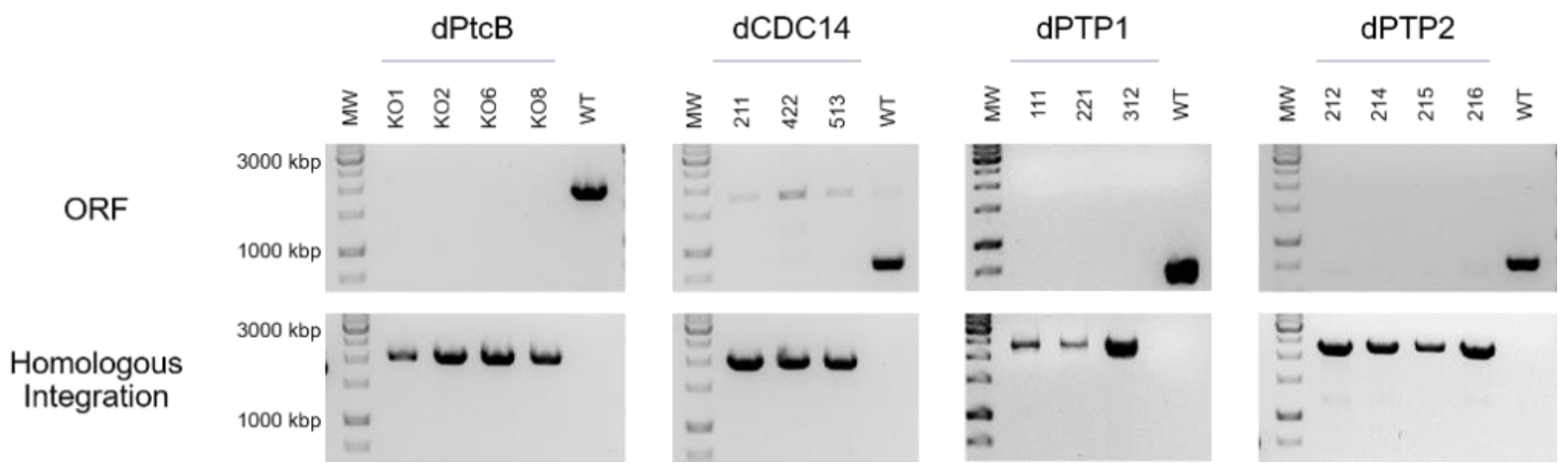

Figure 2. PCR verification of the gene replacement. ORF: amplification of an internal part of the ORF of each gene was performed using primers ORF-FR and ORF-RV. Homologous Integration: a $5^{\prime}$ region of the integrated HYG gene with the upstream flank was amplified, using primers HI-Upstream and NLC37 (Table 1) for the indication of the HYG gene insertion. The numbers indicate independent transformants.

\subsection{Phenotypic Characterization of the Protein Phosphatase Deletion Mutants}

Following the single-conidia isolation, strains that were positive for Hygromycin resistance cassette-homologous integration and negative for the gene of interest were tested for spore phenotypes, growth rates in different types of media, degree of virulence, and the impact of the FA induction on their Hog1 phosphorylation state. These criteria will be described in detail, below, for each of the deletion mutants.

\subsection{Spore and Colony Morphology}

The dPtcB mutants exhibited an abnormal spore phenotype (Figure 3A); this included a shorter spore length (about $20 \mu \mathrm{m}$ in the mutants compared to $100 \mu \mathrm{m}$ in the wild type), a lack of cell compartments in the conidia, and a lower spore count in the mutants (Figure 3A). Furthermore, the colonies had a dramatically altered morphology (Figures 3C, 4 and 5), and were slow growing (Figure 6). The dCDC14 mutants almost completely lacked conidia, and the few conidia present lacked the compartmentation into cells that is seen in WT spores. The spore length ranged from about 50 to $70 \mu \mathrm{m}$ (Figure 3A). Despite the spore structure phenotypes, both the $\mathrm{dPtcB}$ and $\mathrm{dCDC} 14$ spores were able to germinate (an example is shown for dCDC14 in liquid CMX medium, Figure 3B). The dPTP1 and dPTP2 mutants exhibited wild-type growth, spore cell compartments, and colony morphology (Figures $3 \mathrm{~A}$ and 4 ).

The addition of sorbitol to the CM media resulted in longer hyphae compared to CM alone (Figure 5, top panel). From visual inspection, we noted that the hyphae tended to branch to a lesser degree and were generally more invasive, i.e., they grew deeper into the agar (Figure 5, top panel). Although the dHog1 mutants barely grew in the presence of sorbitol, high osmolarity did not promote invasive growth, and the hyphal pattern was essentially normal, with the production of aerial hyphae (Figure 5). The dPtcB mutants showed an altered, disoriented hyphal pattern, and in the presence of sorbitol, the $\mathrm{dPtcB}$ mycelia were highly tumbled, with short branches. The dCDC14 hyphae were tumbled and the colony was less dense, yet it seems that high osmolarity encouraged hyphal branching, even in comparison to the WT. In Figure 5, the minus-FA controls show a slightly different morphology from the minus-sorbitol controls. These differences can be attributed to the presence of DMSO, the solvent for FA. Thus, DMSO caused the hyphae to grow in less density and in disorder, although the radial growth rate was not strongly affected (see Figure 6). The addition of ferulic acid (FA) to the CM media led to increased hyphal density, but with almost normal growth patterns (Figure 5, bottom panel). The dHog1 hyphae were shorter in comparison to the WT. The dCDC14 on DMSO grew as on the CM without DMSO, but again, in the presence of FA, the hyphal growth pattern was similar to the WT (Figure 5) despite the slow growth of dCDC14 (Figure 6). When coping with FA, the colony tends to be denser. The reduction of the linear growth could reflect the ability of the 
fungus to cope with stress. Both dPTP1 and dPTP2 behave as the wild-type when exposed to either sorbitol or FA.

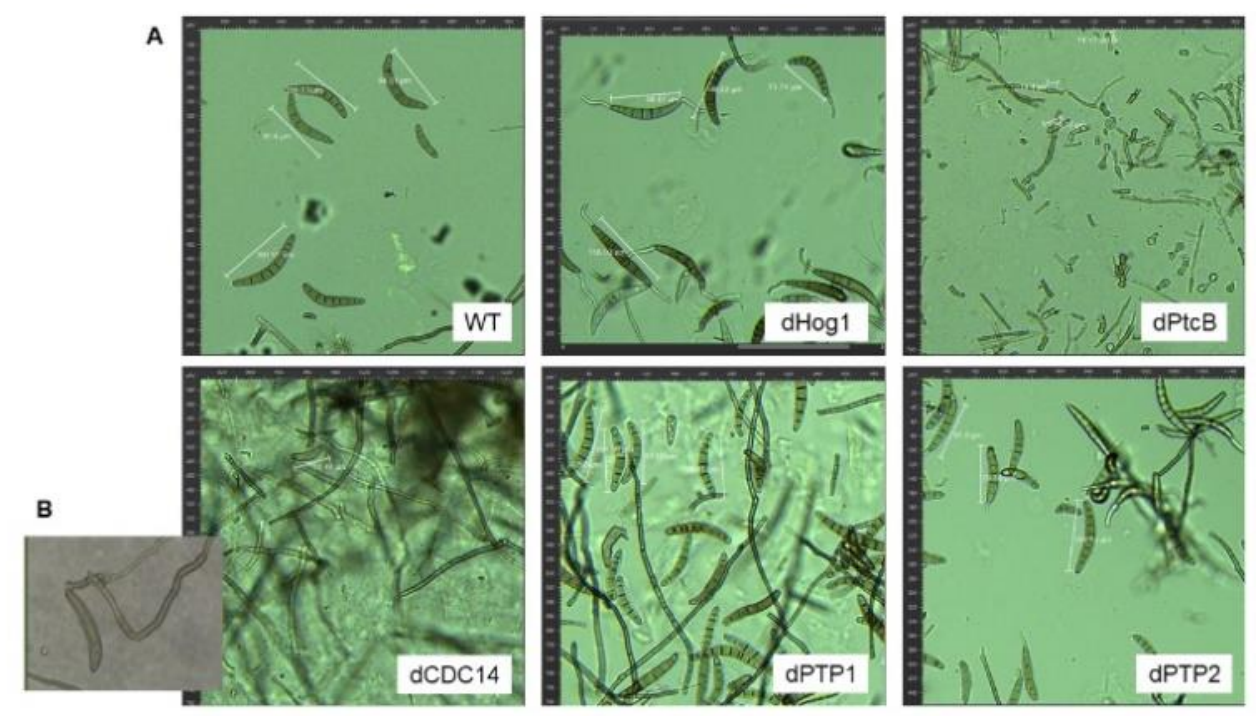

C

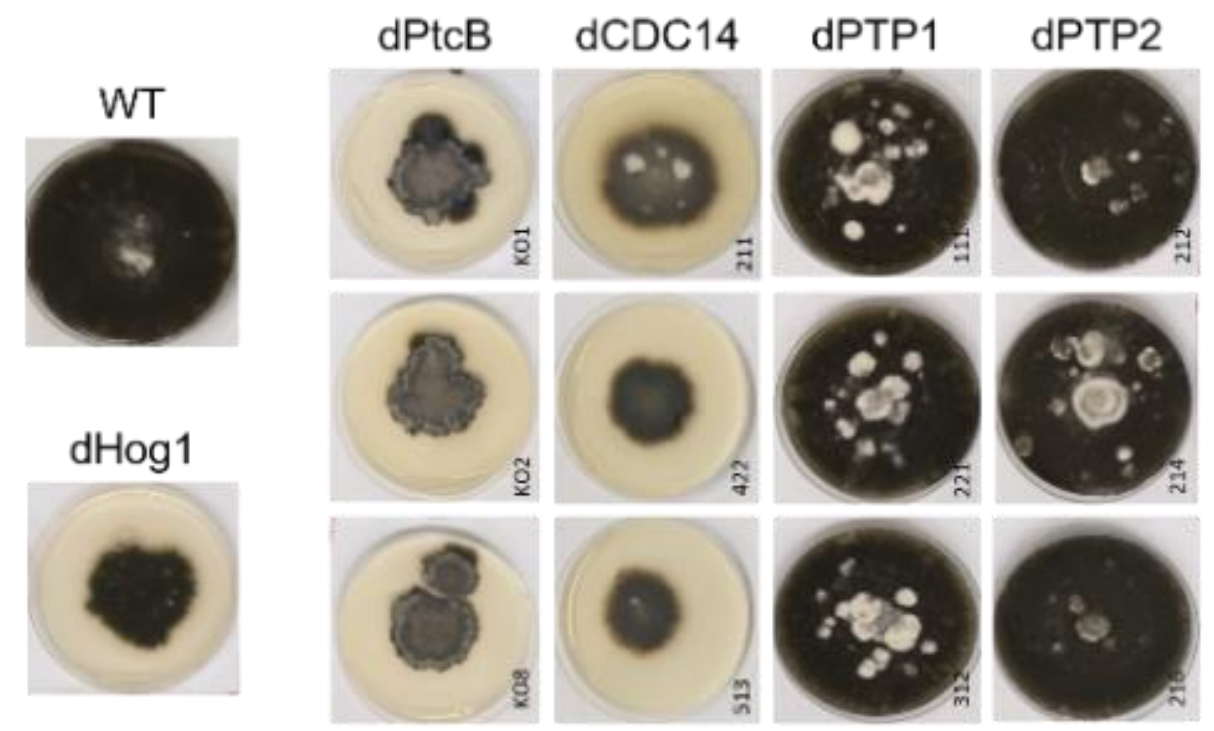

Figure 3. Representative spore (conidia) phenotypes of the WT and mutants. (A) Spores were collected and incubated in CM for $1.5 \mathrm{~h}$ (see Methods). Pictures were taken using a LEICA DMI8 inverted fluorescent microscope. (B) The germination of dCDC14 in a liquid Complete MediumXylose (CMX). The conidiation of dCDC14 is sporadic and rare (panel A, lower left), but the spores that were collected can germinate, as shown in this image. (C) Colony phenotypes of the WT and mutants. From each mutant, at least three independent transformants were grown on CMX for 12 days, and their pictures were taken. 


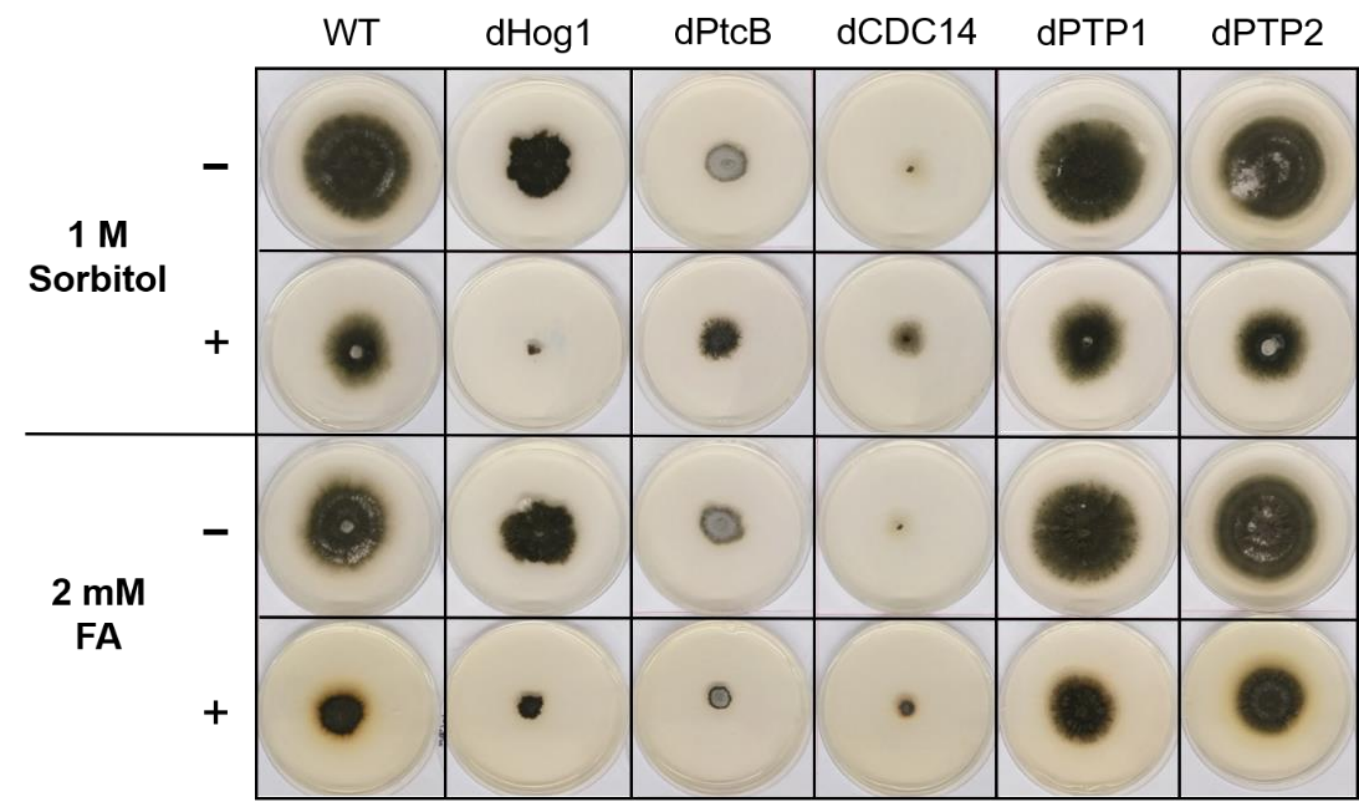

Figure 4. Colony phenotypes under osmotic stress or FA treatment. The cultures were grown for 6 days following their inoculation on $\mathrm{CM}$ agar plates with or without sorbitol ( - or $+1 \mathrm{M}$ sorbitol), or CM with $0.15 \% v / v$ DMSO with or without FA ( - or $+2 \mathrm{mM} \mathrm{FA})$.

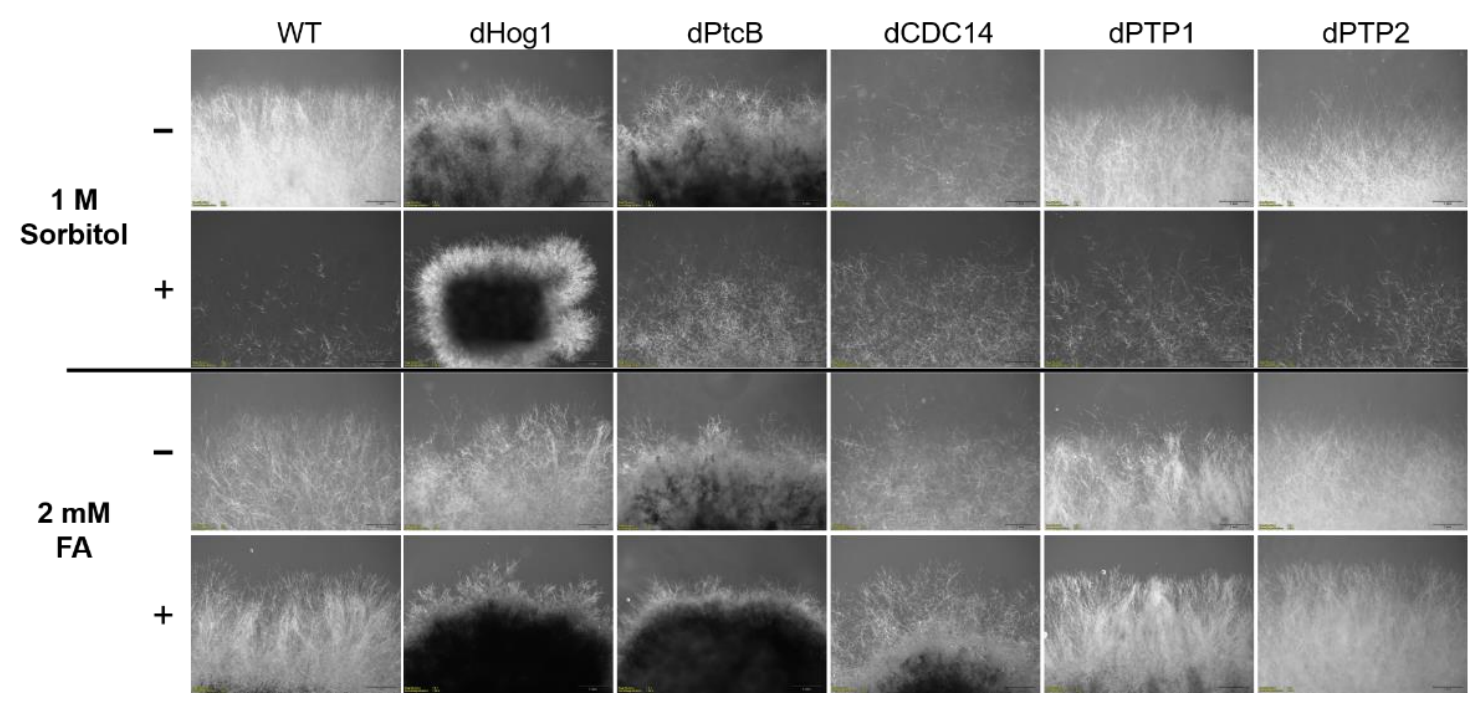

Figure 5. Morphology of the colony margins. The cultures were grown on CM agar plates with or without sorbitol ( - or + $1 \mathrm{M}$ sorbitol), or CM with $0.15 \%$ v/v DMSO with or without FA ( - or $+2 \mathrm{mM} \mathrm{FA}$ ). The pictures were taken 5 days after the inoculum transfer. 


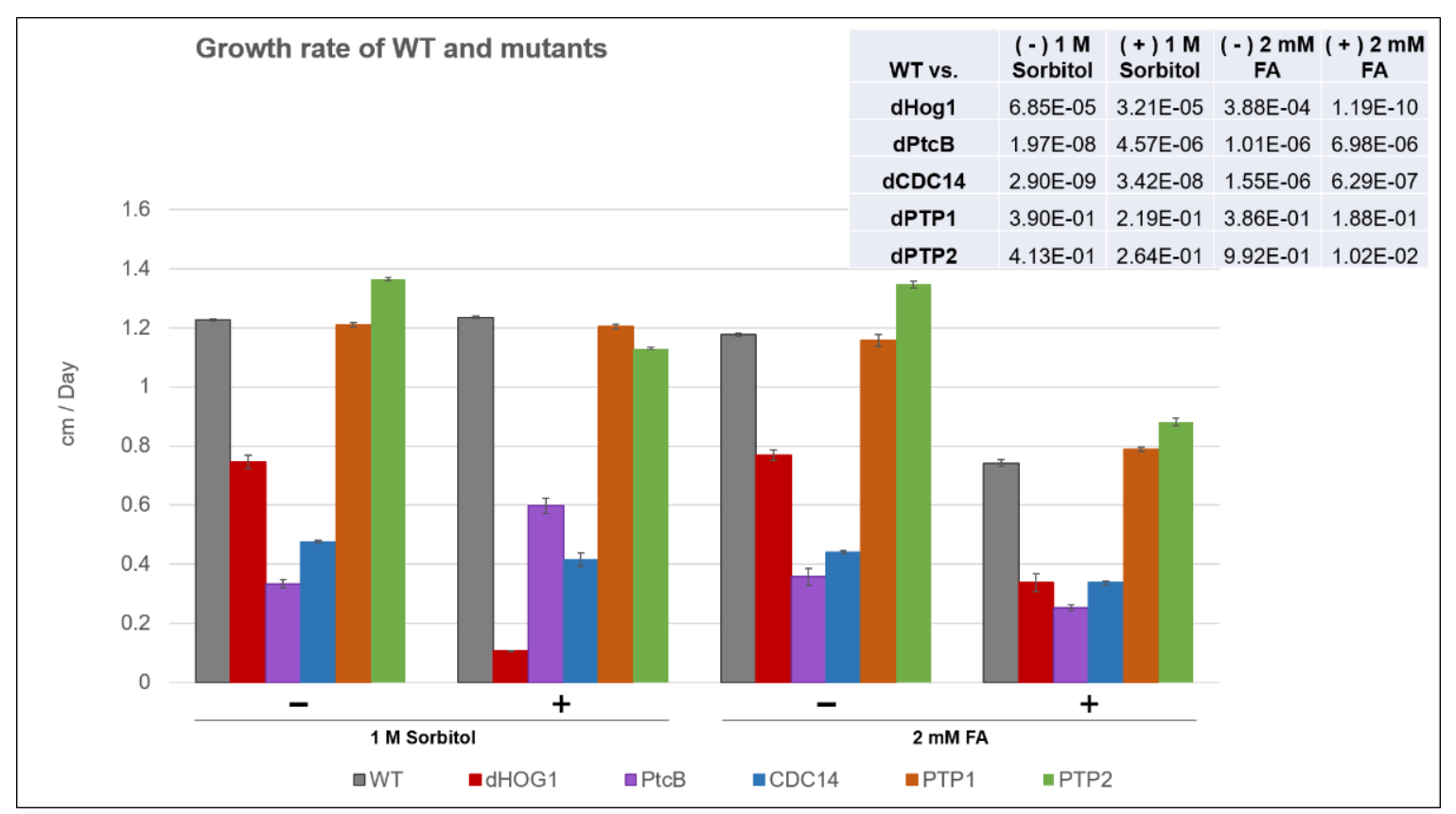

Figure 6. Growth rate of the mutants and WT. The WT and mutants were grown on CM agar plates with or without sorbitol ( - or $+1 \mathrm{M}$ sorbitol), or CM with $0.15 \%$ v/v DMSO, with or without FA ( - or $+2 \mathrm{mM} \mathrm{FA}$ ), for up to $14 \mathrm{~d}$, and the colony radius was measured at intervals (see Methods). The error bars indicate SE. The inset shows the t-test $p$ values for the indicated comparisons.

\subsection{Growth Rate, Germination and Germling Adherence}

The linear radial growth of the WT and mutants is shown in Figure 6 (here and in the following figures, the bar graphs follow a color code identifying each strain). The data are replotted as ratios to show the relative effects of sorbitol and FA (Figure 7). It should be noted that, in some cases, the colony perimeter is not easily visible in Figure 5 due to invasive hyphae; the colony perimeters were marked for measurement at the maximum radius of the colony including invasive hyphae within the agar medium. As can be seen from Figures 6 and 7, along with Figure 4, although DMSO is known to affect cell membranes [31], at the low concentration $(0.15 \%)$ used to apply the FA, it did not affect the radial growth rate of any of the strains tested. Moreover, in the absence of functioning Hog1, high osmolarity strongly decreased the growth rate, as expected [32]. In the presence of FA, the WT growth rate decreases (Figure 7). On the other hand, it was interesting to see that in the WT, spore morphology, attachment, and germination were highly impaired in the presence of high osmolarity, but very much less affected by the presence of FA. Additionally, as expected, $\mathrm{dHog} 1$ had difficulty growing at a high osmolarity. Relative to WT, $\mathrm{dHog} 1$ grew better in the presence of FA than at a high osmolarity, suggesting different pathways for high osmolarity and FA responses, though both affect Hog1's dual phosphorylation. The variability in spore germination and attachment was high (Figure 8A), but the general trend may indicate, again, better attachment and germination in the presence of FA than sorbitol. The $\mathrm{dPtcB}$ and $\mathrm{dCDC} 14$ mutants showed a slower growth rate compared to the WT. Strikingly, $\mathrm{dPtcB}$ grew better on $\mathrm{CM}+$ sorbitol than on $\mathrm{CM}$. Thus, $\mathrm{dPtcB}$ had a relatively better ability to cope with high osmolarity than with the presence of FA (Figures $4 \mathrm{~B}$ and 6 ). In this sense, dHog1 and $\mathrm{dPtcB}$ have opposite stress sensitivities (Figure 7). The attachment and germination of $\mathrm{dPtcB}$ conidia were highly variable and generally impaired (Figure 8). The impaired germination is in agreement with the data for the PtcB ortholog of A. fumigatus [1]. The germination and attachment rates could not be measured for CDC14 due its low spore count; however, as noted above, the few spores formed were able to germinate. The deletion of PTP1 did not affect the growth rate, in accordance with previous reports [27]. dPTP2 showed a trend 
toward a slightly increased growth rate on all media except for CM+sorbitol; the difference was significant only in the presence of FA (Figure 6).

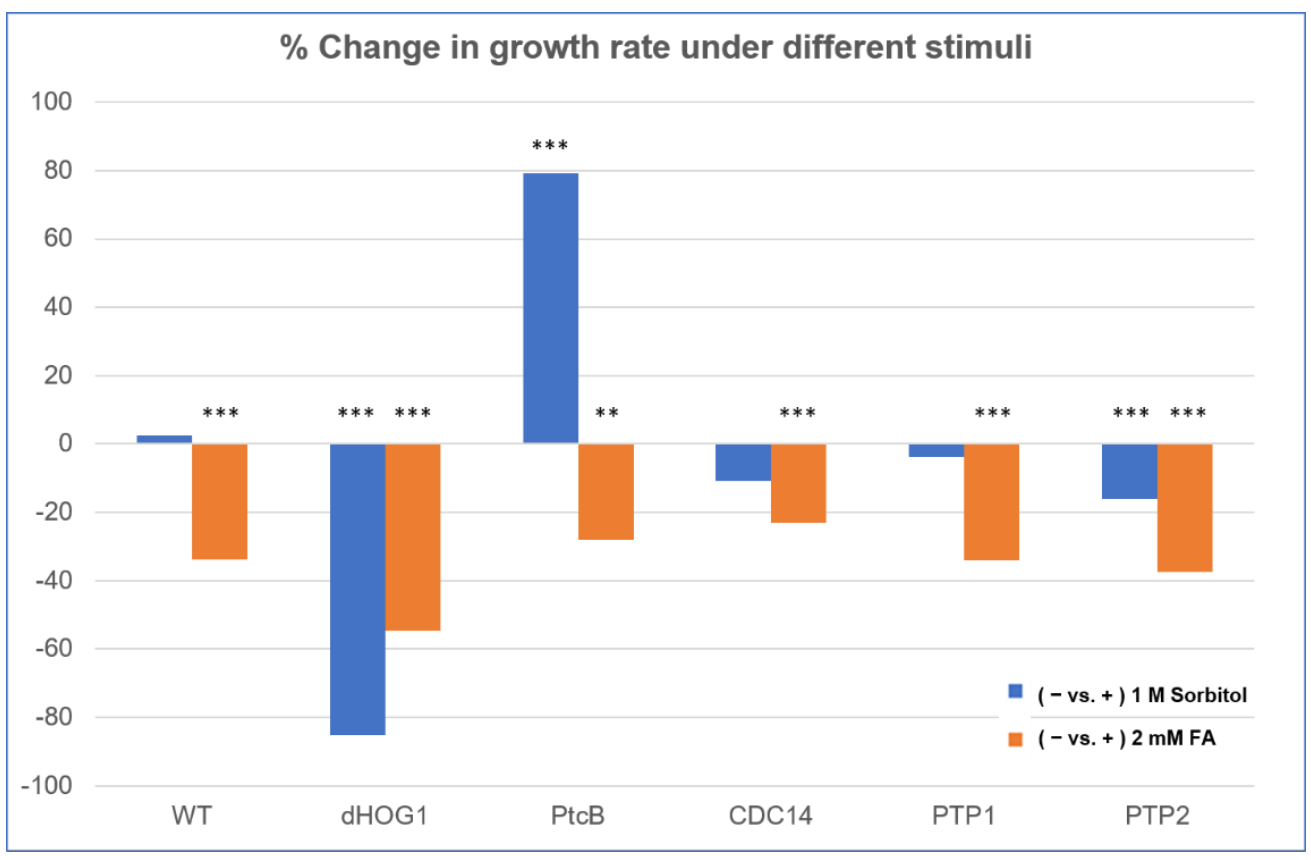

Figure 7. Comparison of FA and sorbitol's effects on the different strains. The data from Figure 6 are replotted as ratios. CM agar plates with or without sorbitol ( - or $+1 \mathrm{M}$ sorbitol) or CM with $0.15 \%$ v/v DMSO with or without FA $(-$ or $+2 \mathrm{mM}$ FA) were compared. The $t$-test $p$-values are marked as follows: $p$-value $<0.05$ — $^{* *} ; p$-value $<0.01$ —** $^{* *}$.

\subsection{Virulence Assays}

The deletion of either PtcB or CDC14 strongly suppressed virulence (Figure 9 and Figure S4), as reported for dPtcb in A. fumigatus on a mammalian host [1], implying a conserved loss of pathogen fitness. Though the growth rate of both the APTP1 and dPTP2 mutants on $\mathrm{CM}$ was not significantly higher than the WT, these mutants were significantly more virulent than the WT, indicating additional factors in the virulence of the mutants compared to WT, other than simply the effects of slower or faster growth rates.

\subsection{The Effect of Phosphatase Deletions on Hog1 Dual Phosphorylation}

In order to explore the involvement of these genes in the Hog1 phosphorylation levels in response to FA, the mutants and WT were given a brief exposure to FA. The phosphorylation was assayed using immunoblots with an antibody to dual-phosphorylated mammalian P38 (anti p-P38), which has been used extensively to detect dual-phophosphorylated Hog1 (pHog1) in fungi (for example, [33,34]). An antibody against yeast Hog1 (anti Hog1) was used to quantify the total Hog1 protein levels. In the WT, the addition of FA resulted in decreased ppHog1 levels (Figure 10A). This was not the case, however, for two of the mutants; in the strains deleted for PtcB or CDC14, there was little or no reduction in the ppHog1 levels upon exposure to FA (Figure 10A). The addition of sorbitol to the CM media rapidly increased the ppHog1 levels, in the WT and in all four mutants (Figure 10B). Two points emerge from these data: first, the modulation of Hog1 dual phosphorylation by FA and sorbitol are not only opposite (Figure 10A,B) but are also genetically separable, because the loss of PtcB or CDC14 affects the response to FA but not to high osmolarity (sorbitol). The second point is that two distinct phosphatases belonging to different classes are both required for dephosphorylation in response to FA (Figure 10A). This suggests multiple modulators for Hog1, but because each phosphatase mutant showed different properties, the question of the mechanism of Hog1 dephosphorylation upon exposure to FA arises. Next, we tested the effect of sequentially adding FA and sorbitol to the CM media. As 
shown in Figure 10C, the addition of sorbitol partially counteracted the decrease in the phosphorylation levels due to FA exposure.
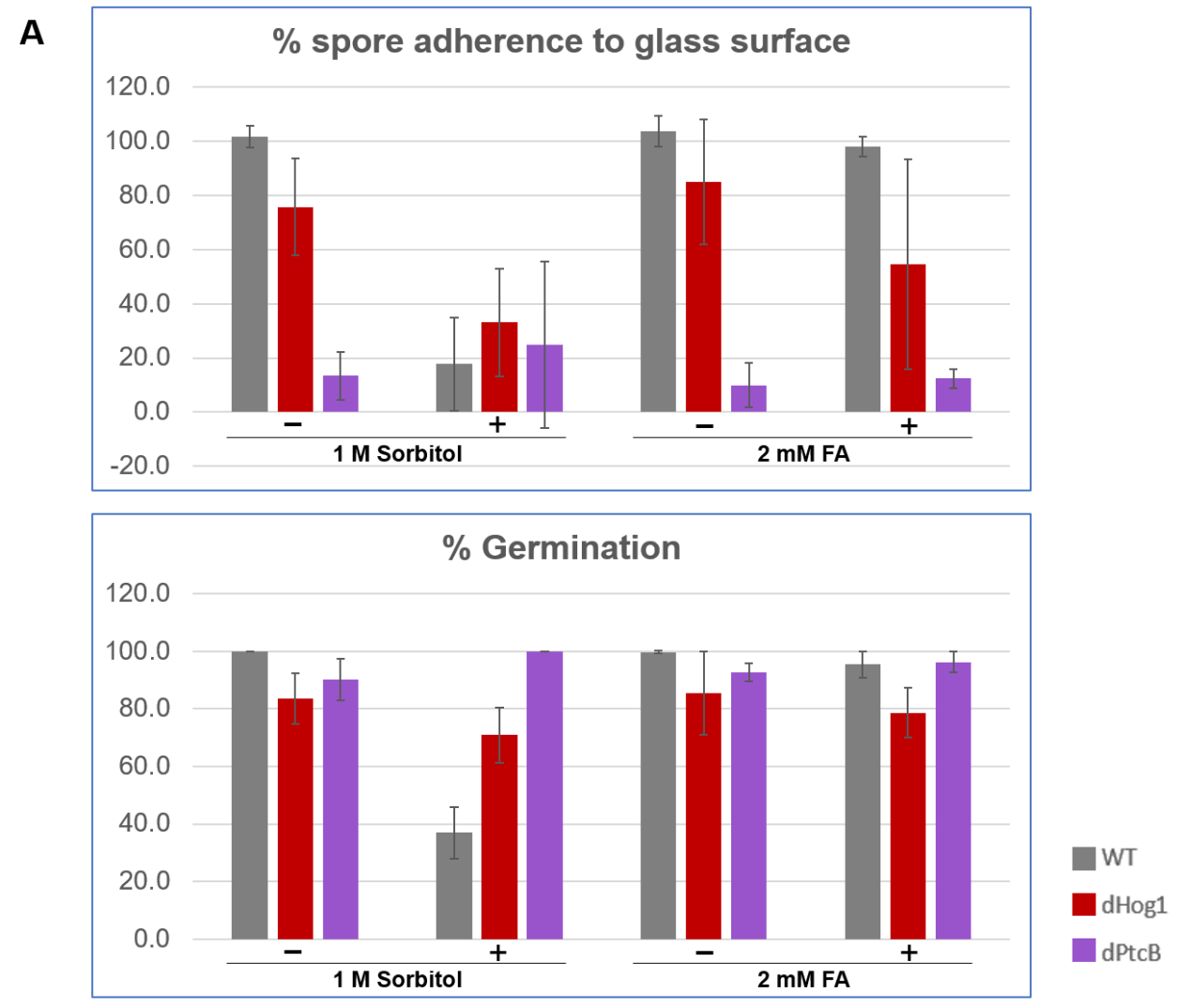

B

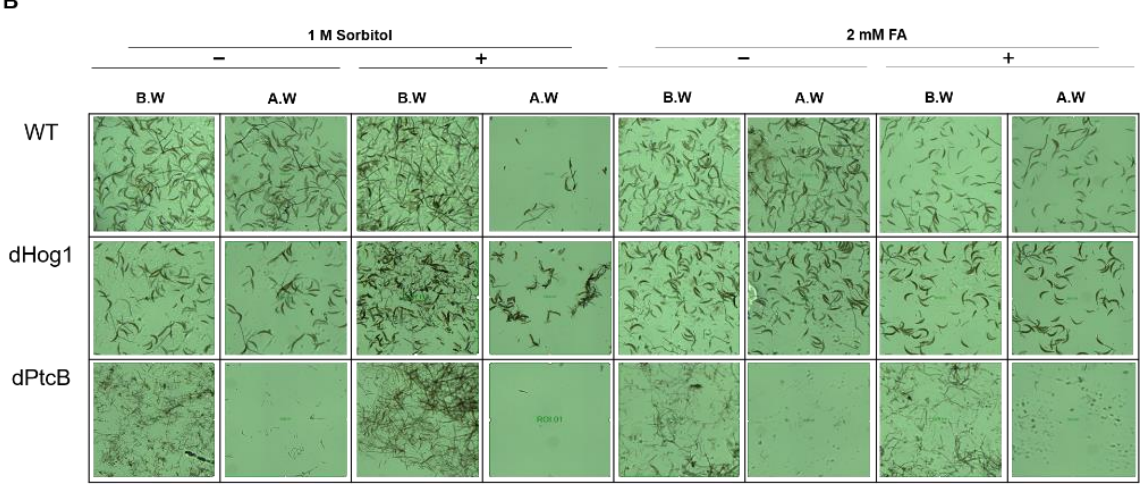

Figure 8. Spore attachment and germination of strains on stress-inducing growth media. (A) The percentage of the spore attachment and germination. C. heterostrophus $\mathrm{C} 4 \mathrm{WT}$ strain and mutants were grown in CM agar plates with or without sorbitol ( - or $+1 \mathrm{M}$ sorbitol), or CM with $0.15 \%$ v/v DMSO with or without FA ( - or +2 mM FA), for $14 \mathrm{~d}$ ( $8 \mathrm{~h} / 16 \mathrm{~h}$ dark/light cycles). Spore suspensions were collected from each, and were incubated on a cover slip in a humid chamber for $1.5 \mathrm{~h}$ (see Methods). The pictures were taken using a Leica DMI8 inverted microscope. Each droplet was washed twice with $50 \mu \mathrm{L}$ DDW and documented again. The image processing was performed using the LASX program. Standard deviation bars were added. (B) Representative pictures corresponding to the treatments in panel A. 
A $\quad$ WT dHog1

$\mathrm{dPtcB}$

dCDC14

dPTP1

dPTP2
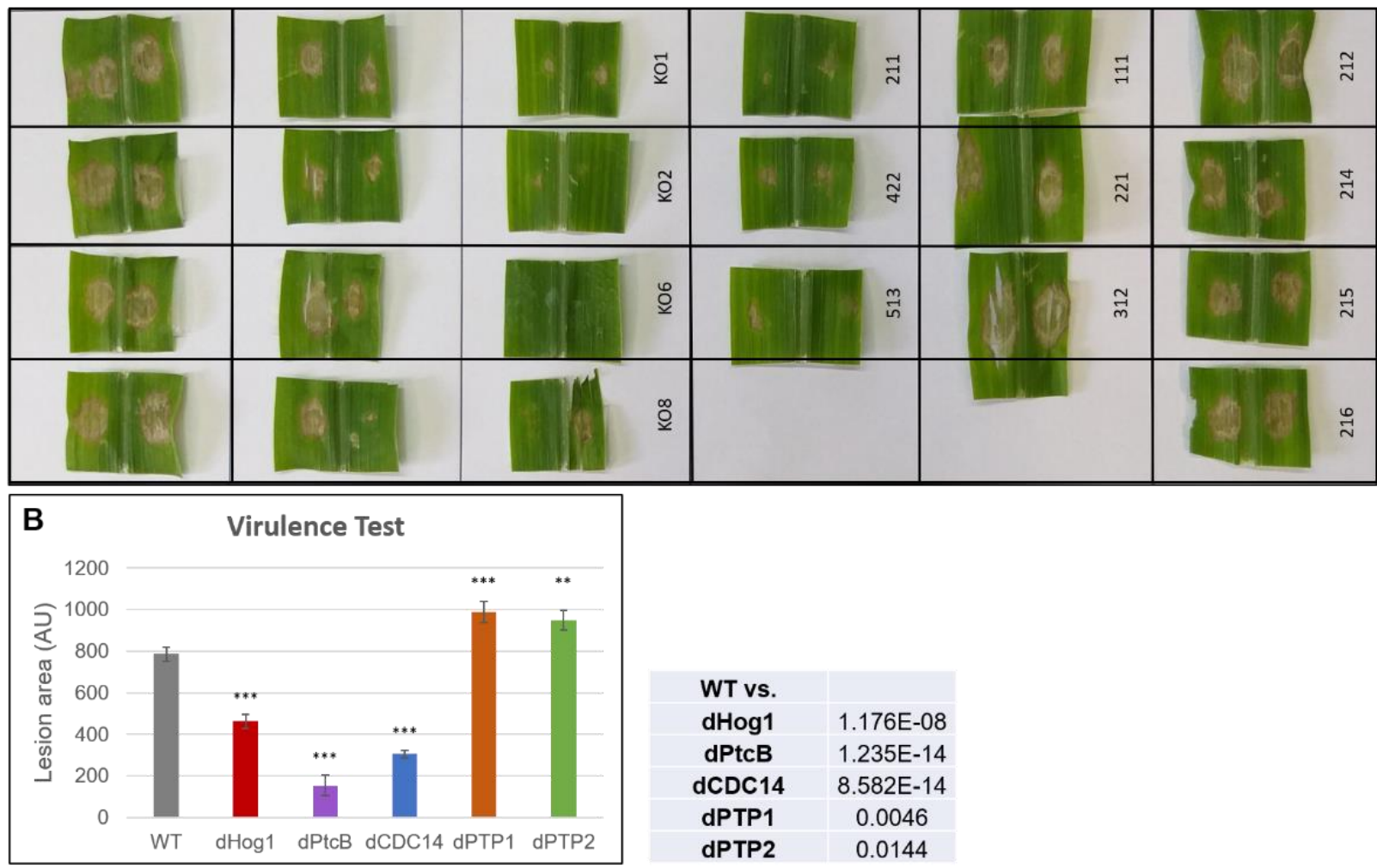

\begin{tabular}{c|c|}
\hline WT vs. & \\
\hline dHog1 & $1.176 \mathrm{E}-08$ \\
\hline dPtcB & $1.235 \mathrm{E}-14$ \\
\hline dCDC14 & $8.582 \mathrm{E}-14$ \\
\hline dPTP1 & 0.0046 \\
\hline dPTP2 & 0.0144
\end{tabular}

Figure 9. Virulence assay of mutants and WT on maize leaves. (A) The third leaf of corn plants (the rounded, juvenile leaf was counted as leaf 'zero') was infected with a $\sim 0.09 \mathrm{~cm}^{2}$ agar block inoculum for $24 \mathrm{~h}$, and was then removed (see Methods). The lesions were measured 4 days from infection. (B) Quantitation of the lesion areas. The lesion areas were measured using ImageJ. The graph represents the average lesion area of each strain, calculated from 3 biological repeats for each independent transformant (for the photos of all of the lesions analyzed, see Supplementary Figure S2). The error bars indicate SE. The $t$-test $p$-values of each strain vs. the WT were calculated and are marked as follows (inset): $p$-value $<$ 0.05 - $^{* *} ; p$-value $<0.01$ —*** $^{* *}$.

This shows that the effects are opposite and additive, though the question of the biochemical mechanism cannot yet be resolved. Finally, we studied the time course of the phosphorylated Hog1's appearance following its exposure to FA and sorbitol, up to $30 \mathrm{~min}$ after its exposure to these compounds. As shown in Figure 10D, pHog1 accumulated gradually over time following the exposure to sorbitol. In contrast, $\mathrm{pHog} 1$ was dramatically and rapidly reduced following the exposure to FA. Both inducers act in a time frame that is consistent with cellular signaling cascades, but the time course indicates rapid and efficient dephosphorylation, compared to the somewhat-slower phosphorylating activity of the upstream MAPKK. The low molecular weight bands observed (Figure 10C) probably reflect partial proteolytic degradation products of Hog1 (see Figure 5 in [35]); such bands were not observed in the dHog1 mutants, in line with this hypothesis (Roni Koren, unpublished observations). 

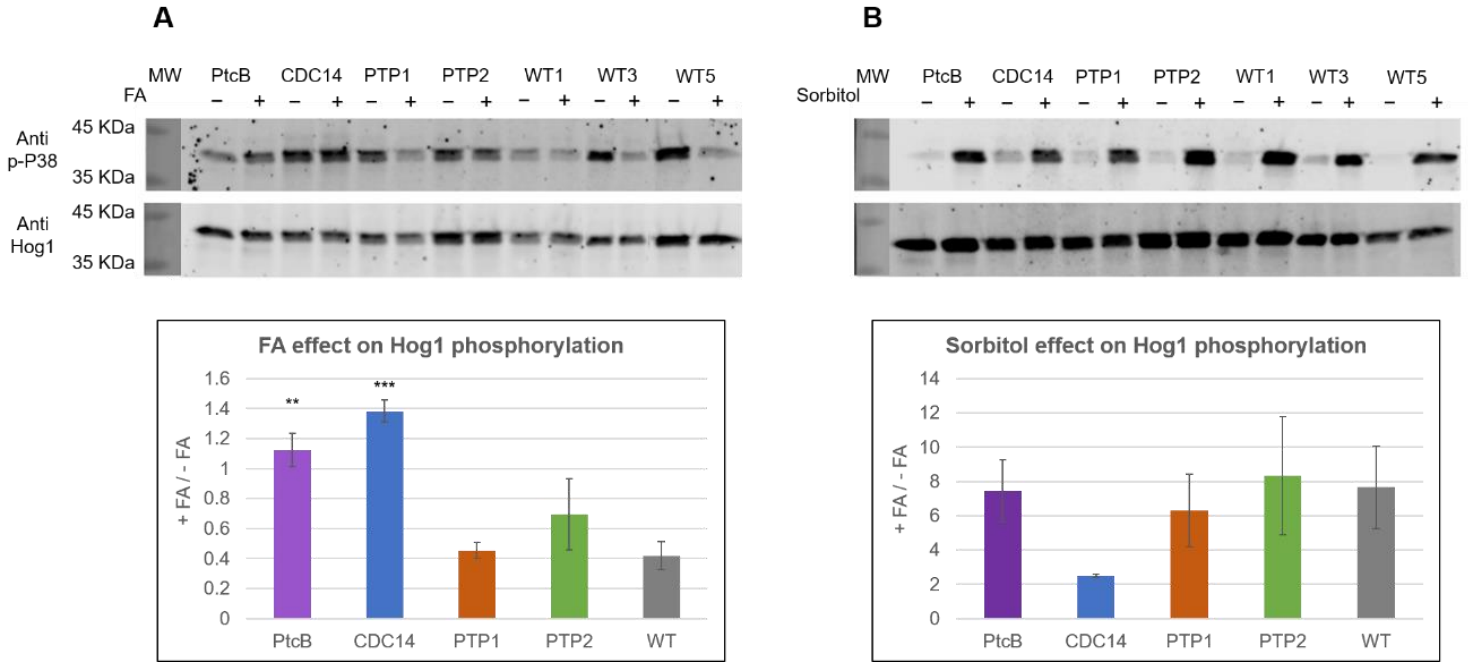

C

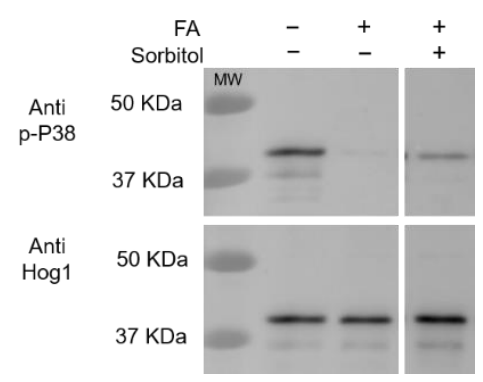

D

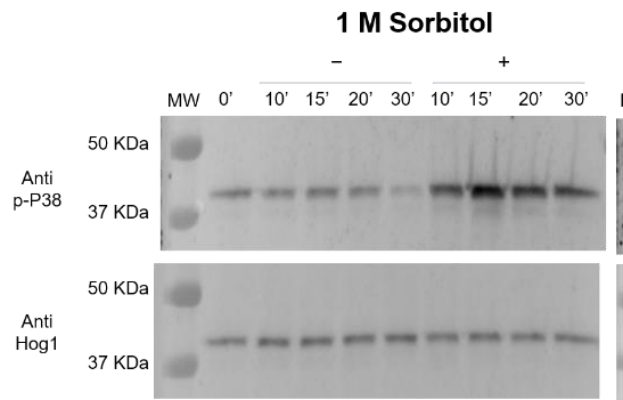

2 mM FA
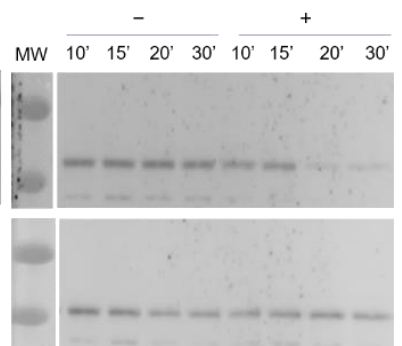

Figure 10. FA and sorbitol's effects on Hog1 dual phosphorylation in WT and phosphatase mutants, assayed by immunoblotting. (A) FA, (-) indicates CM with $0.15 \%$ v/v DMSO (control); (+) indicates the addition of $2 \mathrm{mM}$ FA. (B) Sorbitol, (-) indicates $\mathrm{CM} ;(+)$ indicates the addition of $1 \mathrm{M}$ sorbitol. Blots of the total protein extract of the WT and the different mutants were probed with either a p-P38 antibody (Cell Signaling, T180-Y182, 9211S) or a Hog1 antibody (Santa Cruz, y-215, sc-9079). Representative blots are shown (for the complete data set, see supplementary Figure S5). The graphs represent the ratio change in the signal upon induction (calculated from three independent deletion transformants, indicated by numbers labelling each lane). The quantitation was performed using Image J. The $t$-test $p$-values of each strain vs. the WT were calculated, and are marked as follows: $p$-value $<0.05-^{* *} ; p$-value $<0.01-^{* * *}$. (C) The sequential effect of FA and osmotic stress on Hog1 in WT. The samples were analyzed as in panels (A,B). C. heterostrophus C4 WT strain protein extract, from CM with $0.15 \% v / v$ DMSO (-/-), CM with $0.15 \%$ v/v DMSO with $2 \mathrm{mM} \mathrm{FA} \mathrm{(+/-),} \mathrm{and} \mathrm{CM} \mathrm{with} 0.15 \%$ v/v DMSO with $2 \mathrm{mM}$ FA, with the sequential addition of sorbitol to $1 \mathrm{M}$ concentration, as described in the method section $(+/+)$. In this panel, different parts of the same blot are shown side by side at the same scale, and this is indicated by a space between the sections. (D) Time course for the sorbitol and FA induction. The media and immunoblotting were as they were in panels $(\mathbf{A}, \mathbf{B})$.

\section{Discussion}

\subsection{Hog1 Dephosphorylation as a Signal}

Ferulic acid (FA), an abundant plant phenolic, acts as a stress signal in the maize pathogen C. heterostrophus, triggering cell death [8] and regulating the transcript levels of a large number of genes [16]. Our observation that exposure to FA leads to the dephosphorylation of an initial, basal level of TXY dual phosphorylation [8] could have (at least) two functional explanations: (1) dephosphorylation, by lowering the activation level of Hog1, could transduce information, for example by lowering the level of an inhibitory transcription factor; (2) the sustained activation of Hog1 can promote cell death in fungi, so that dephosphorylation could serve as a mechanism to allow the cell to mitigate the celldeath-promoting effect of FA. Supporting this possibility, an earlier study in Cryptococcus neoformans serotype A H99 [36] showed the constitutive phosphorylation of Hog1, and 
dephosphorylation upon exposure to high osmolarity. Both putative functions (1) and (2) of the rapid dephosphorylation of Hog1 are thus of interest, and as a first step toward a mechanistic understanding, we identified protein phosphatase candidates. Mutants were constructed in four candidate genes: PtcB, CDC14, PTP1 and PTP2. The results show that, in addition to modulating the Hog1 phosphorylation level, the loss of phosphatases has profound developmental phenotypes. Viable mutants were obtained for all four genes, and at least three independent transformants were verified and analyzed for all of the phenotypes shown. Furthermore, the introduction of a WT copy rescued the phenotypes of $\mathrm{dPtcB}$. PtcB and the CDC14 mutants show a decreased growth rate, while the tyrosine phosphatases PTP1,2 are, for the most part, dispensable for normal growth and virulence. The loss of PtcB has a more drastic effect on colony morphology and growth than CDC14, but CDC14 has an almost equally drastic reduction in virulence (Figures 4-9). While loss-of-function mutations are generally expressed as negative phenotypes, an interesting exception was seen for $\mathrm{dPtcB}$ following its exposure to sorbitol (CMS, Figure 4), where $\mathrm{dPtcB}$ shows increased, rather than decreased, growth compared to the control. This is further emphasized in Figure 7, where the loss of PtcB has opposite effects on sorbitol or FA-containing media to those seen for the other mutants. This is consistent with PtcB being a major modulator of the Hog1-mediated stress response, in agreement with prior findings in A. fumigatus [1]. The opposite effects of sorbitol and FA stresses (Figure 7) suggest that distinct PtcB-dependent biochemical pathways are at work in tuning the level of Hog1 activation. The MAP kinase Hog1, and the stress signals that it transduces, are important in fungal development and virulence. A question thus arises as to why in the case of high osmolarity or exposure to fludioxonil, the fungus 'fights' regulated cell death (RCD) by increasing Hog1 phosphorylation levels, while upon exposure to FA, the Hog1 phosphorylation levels instead decrease. The biochemical underpinnings of these opposite responses will be a subject for future work.

\subsection{Contribution of More than One Phosphatase to FA-Induced Hog1 Dephosphorylation}

The loss of either PtcB or CDC14 compromises the full WT regulation of phospho TXY Hog1 levels (Figure 10A,B). There is a trend (though not statistically significant) suggesting that PTP2 might be considered as well: dephosphorylation in response to FA appears to be reduced in this mutant (Figure 10A). Prior work in Neurospora and Aspergillus $[16,18]$ is in line with our observation that more than a single phosphatase acts on Hog1. PtcB andCDC14 (and perhaps PTP2) participate in the dephosphorylation of phospho-Hog1. One explanation may be sequential activity, in which the action of one phosphatase is needed for a second to act. There is a report that mammalian MAPKs, starting in their non-activated state, are phosphorylated first on tyrosine. Upon induction, the tyrosinephosphorylation level accumulates to a threshold which, once reached, phosphorylation on threonine is added, and the MAPK — in its dual phosphorylated form-is active [37]. This property of a mammalian MAPK raises the possibility that dephosphorylation could, likewise, be sequential, rather than concerted, with the activity of one phosphatase setting the stage for a second one to act. Mutants lacking either would then show a defect in the dephosphorylation of the TXY motif. This general question can be investigated by timeresolved phosphoprotein MS/MS experiments in mammalian cells, yeast, or filamentous fungi. Another explanation could be the indirect developmental consequences of the lack of a particular phosphatase on the activity of others.

\subsection{Hog1 Phosphatases of C. heterostrophus in Comparison to Other Fungal Models}

The developmental roles for the four phosphatase genes studied here have been reported in other fungi. Fungal signaling pathways based on conserved components are nevertheless 'wired' differently in diverse species. The PtcB of A. fumigatus is involved in attachment and virulence. It also had a remarkable effect on the cell surface, conidia and germling adhesion, biofilm formation, and MpkA phosphorylation, and its involvement in the CWI pathway was also suggested. Furthermore, a dPtcB strain showed impaired 
virulence in a mouse model for invasive pulmonary aspergillosis [1]. In S. cerevisiae, in contrast, Hog1 dephosphorylation is regulated by phosphotyrosine phosphatases (Ptp2 and Ptp3) or phospho S/T phosphatases (Ptc1). This is also in accordance with the Ptc or Ptp-types of phosphatases in the pathogenic basidiomycete yeast, C. neoformans [27]. Collectively, the existing literature suggests that PtcB is a central Hog1 phosphatase, and its loss affects a wide variety of cellular processes. Generally, PtcB is discussed in cases of high osmolarity causing high $\mathrm{pHog} 1$ levels, yet in our case, PtcB was involved in lowering the pHog1 levels below the basal level. This is a novel role for both PtcB and Hog1.

Early studies in yeast showed that CDC14 plays a key role in regulating late mitotic processes [38]. More recently, however, studies in some filamentous species have revealed that CDC14 is not always an essential gene, and also that it is involved in diverse cellular functions. The deletion of CDC14 in Metarhizium acridum, for example, resulted in a defective growth phenotype and multiple nuclei in each hyphal compartment, indicating nuclear division and cytokinesis defects as expected, yet without significant effects on the sensitivity to cell wall damage reagents, or sensitivity to osmotic and oxidative stress [22]. On the other hand, the inactivation of CDC14 in Beauveria bassiana resulted in hypersensitivity to oxidative, osmotic, cell wall and mitosis-perturbing stresses [24]. Filamentous fungal orthologs of yeast CDC14 could have previously overlooked functions in multistress responses, including the possible interactions of CDC14 with other phosphatases and MAPKs. Our findings in C. heterostrophus support this view.

Two protein tyrosine phosphatases (PTPs) were chosen here as candidate Hog1 phosphatases because they are known to modulate the Hog1 phosphorylation levels in several species. The HOG1 phosphorylation levels decreased in Magnaporthe oryzae PTP2overexpressing strains, while PTP2 loss-of function mutants had higher phosphorylation levels of HOG1 compared to the WT [15]. The deletion of PTP2 and PTP3 (these two genes may have redundant functions) affected the sporulation in yeast, where the deletion of these genes blocks cells at an early stage of sporulation before premeiotic DNA synthesis and the induction of meiotic-specific genes [39]. Moreover, PTP2 was shown to be involved in mediating vegetative growth, sexual differentiation, stress responses, anti-fungal drug resistance, and virulence factor regulation in C. neoformans through the negative-feedback loop of the Hog1 pathway, while PTP1 was not essential for Hog1 regulation [27]. PTP1 was dispensable despite its Hog1-dependent induction. Interestingly, the loss of PTP2 did not compromise viability, since PTP1 served as a complementary PTP to control some stress responses [24]. In C. heterostrophus, the lack of PTP1 and PTP2 in our mutant strains lead to no obvious phenotypes and little, if any, effect on the steady-state basal Hog1 phosphorylation levels or the dephosphorylation of Hog1 in response to FA. This finding again underscores the differences between the species, but should also encourage the construction of double mutants and over-expression strains in future work.

\section{Conclusions}

Hog1 is well-studied in the context of high osmolarity stress, but the FA-induced pathway studied here has revealed new insights regarding the roles of Hog1 in pathogenicity and in developmental pathways. The results indicate a complex relationship between Hog1 dephosphorylation and the response of the fungal cell to chemical stress by plant phenolics, and a multi-phosphatase regulation system of Hog1. Moreover, apparently, the effect of the deletion of a particular tyrosine phosphatase was less critical than a threonine phosphatase. The deletion of PtcB and CDC14 resulted in abnormal phenotypes-including defective spores, a slower growth rate, and decreased virulence-as opposed to the $\mathrm{KO}$ of PTP1 and PTP2. These results open many questions regarding the mechanism by which dephosphorylation is sensitized by FA and the nature of the phosphatase(s) involved. We predict that the sensitivity to FA-induced cell death will be altered when FA-induced Hog1 dephosphorylation is compromised. This prediction can be tested with the help of the mutants described here. Understanding the role of protein phosphatases in the FA-Hog1 
pathway may be of general significance for the fungal pathogens of plants: the osmotic response pathway is highly conserved, and exposure to plant phenolics is widespread.

Supplementary Materials: The following are available online at https:/ /www.mdpi.com/2309-608 X/7/2/83/s1. Figure S1. C. heterostrophus candidate phosphatase-encoding genes. (A) Phylogenetic tree of A. fumigatus phosphatases (Winkelströter et al. [19], reprinted here with permission via RightsLink). Arrows indicate the choice of representatives of three families. (B-D) Following reciprocal BLASTP searches, phylogenetic trees of the predicted $C$. heterostrophus phosphatase candidate ortholog genes were generated using the Phylogeny.fr pipeline (for details see Methods and the legend to Table 2). Each entry in the trees starts with an informative gene name (organism: Nc, Neurospora crassa; Ch, C. heterostrophus; Mus, mouse, Cael, C. elegans, followed by an abbreviated phosphatase class name and, last, the NCBI protein database ID). Protein domains indicating functional activity of each phosphatase and its classification appear below each tree. Figure S2. Analysis of PtcB complemented strains (AddBack, AB). (A) PCR verification of gene replacement. ORF- Amplification of the full ORF of the PtcB gene was performed using primers ORF-FR \& ORF-RV. Homologous Integration $-5^{\prime}$ region of the integrated HYG gene with upstream flank was amplified, using primers HI-Upstream and NLC37 (Table 1) for the detection of the original HYG gene insertion. In some panels different parts of the same gel are shown side by side at the same scale, and this is indicated by a space between sections. (B) WT vs. $\mathrm{dPtcB} A B$ colony phenotype. (C) WT vs. PtcB AB growth rate. WT was grown on $\mathrm{CMX}$ agar plates. PtcB AB was grown both on CMX agar plates containing HYG $(100 \mu \mathrm{g} / \mathrm{mL})$ and CMX agar plates containing HYG $(100 \mu \mathrm{g} / \mathrm{mL})$ and neomycin $(1 \mathrm{mg} / \mathrm{mL})$. (D) Virulence assay of WT vs. dPtcB AB was performed as in Figure 9. (E-F). Effect of FA induction on Hog1 phosphorylation levels in the WT vs. PtcB AB was assayed as in Figure 10A, B. Figure S3. Quantitation of lesion areas from the virulence assays of PtcB AddBack and WT on maize leaves. Methods were as for Figure 9. Figure S4. Quantitation of lesion areas from virulence assay of mutants and WT on maize leaves. Methods were as for Figure 9. Strain M6 (top left) is not related to this study. Figure S5. FA and sorbitol effect on Hog1 dual phosphorylation in WT and phosphatase mutants, assayed by immunoblotting. All biological repeats for Figure 10: blots of total protein extract of WT and the different mutants, with (+) or without (-) sorbitol or FA induction.

Author Contributions: R.Z. and R.K. performed the experimental work, B.A.H. designed the approach, and R.Z. and B.A.H. drafted the manuscript. All authors have read and agreed to the published version of the manuscript.

Funding: Funded by ISF 420/17 from the Israel Science Foundation.

Institutional Review Board Statement: Not applicable.

Informed Consent Statement: Not applicable.

Data Availability Statement: All data are available in the manuscript and accompanying supplementary materials.

Acknowledgments: We thank Laurie D. Cohen for critical comments and for editing the manuscript. pNG was kindly provided by B. Gillian Turgeon. This study was funded by ISF $420 / 17$ from the Israel Science Foundation. R.Z. gratefully acknowledges a predoctoral fellowship from the Irwin and Joan Jacobs Graduate School, Technion, and a MSc/PhD fellowship from the Israel Scholarship Education Foundation (ISEF).

Conflicts of Interest: The authors declare no conflict of interest. The funders had no role in the design of the study; in the collection, analyses, or interpretation of data; in the writing of the manuscript, or in the decision to publish the results.

\section{References}

1. Winkelströter, L.K.; Bom, V.L.P.; de Castro, P.A.; Ramalho, L.N.Z.; Goldman, M.H.S.; Brown, N.A.; Rajendran, R.; Ramage, G.; Bovier, E.; dos Reis, T.F.; et al. High osmolarity glycerol response PtcB phosphatase is important for Aspergillus fumigatus virulence. Mol. Microbiol. 2015, 96, 42-54. [CrossRef] [PubMed]

2. Cheng, H.C.; Qi, R.Z.; Paudel, H.; Zhu, H.J. Regulation and function of protein kinases and phosphatases. Enzym. Res. 2011, 2011. [CrossRef] [PubMed]

3. Mattison, C.P.; Ota, I.M. Two protein tyrosine phosphatases, Ptp2 and Ptp3, modulate the subcellular localization of the Hog1 MAP kinase in yeast. Genes Dev. 2000, 14, 1229-1235. [PubMed] 
4. Peterson, R.T.; Desai, B.N.; Hardwick, J.S.; Schreiber, S.L. Protein phosphatase 2A interacts with the 70-kDa S6 kinase and is activated by inhibition of FKBP12-rapamycin-associated protein. Proc. Natl. Acad. Sci. USA 1999, 96, 4438-4442. [CrossRef] [PubMed]

5. Ayllón, V.; Martínez-A, C.; García, A.; Cayla, X.; Rebollo, A. Protein phosphatase $1 \alpha$ is a Ras-activated Bad phosphatase that regulates interleukin-2 deprivation-induced apoptosis. EMBO J. 2000, 19, 2237-2246.

6. Kim, J.; Oh, J.; Sung, G.-H. MAP kinase Hog1 regulates metabolic changes induced by hyperosmotic stress. Front. Microbiol. 2016. [CrossRef]

7. Hamel, L.P.; Nicole, M.C.; Duplessis, S.; Ellis, B.E. Mitogen-activated protein kinase signaling in plant-interacting fungi: Distinct messages from conserved messengers. Plant Cell 2012, 24, 1327-1351. [CrossRef]

8. Shalaby, S.; Larkov, O.; Lamdan, N.L.; Goldshmidt-Tran, O.; Horwitz, B.A. Plant phenolic acids induce programmed cell death of a fungal pathogen: MAPK signaling and survival of Cochliobolus heterostrophus. Environ. Microbiol. 2016, 18, 4188-4199. [CrossRef]

9. Lygin, A.V.; Upton, J.; Dohleman, F.G.; Juvik, J.; Zabotina, O.A.; Widholm, J.M.; Lozovaya, V.V. Composition of cell wall phenolics and polysaccharides of the potential bioenergy crop -Miscanthus. GCB Bioenergy 2011, 3, 333-345. [CrossRef]

10. De Oliveira, D.M.; Finger-Teixeira, A.; Rodrigues Mota, T.; Salvador, V.H.; Moreira-Vilar, F.C.; Correa Molinari, H.B.; Craig Mitchell, R.A.; Marchiosi, R.; Ferrarese-Filho, O.; dos Santos, W.D. Ferulic acid: A key component in grass lignocellulose recalcitrance to hydrolysis. Plant Biotechnol. J. 2015, 13, 1224-1232. [CrossRef]

11. Condon, B.J.; Leng, Y.; Wu, D.; Bushley, K.E.; Ohm, R.A.; Otillar, R.; Martin, J.; Schackwitz, W.; Grimwood, J.; MohdZainudin, N.A.I.; et al. Comparative Genome Structure, Secondary Metabolite, and Effector Coding Capacity across Cochliobolus Pathogens. PLoS Genet. 2013, 9, e1003233. [CrossRef] [PubMed]

12. Shalaby, S.; Horwitz, B.A. Plant phenolic compounds and oxidative stress: Integrated signals in fungal-plant interactions. Curr. Genet. 2015, 61, 347-357. [CrossRef] [PubMed]

13. Vendrell, A.; Martánez-Pastor, M.; González-Novo, A.; Pascual-Ahuir, A.; Sinclair, D.A.; Proft, M.; Posas, F. Sir2 histone deacetylase prevents programmed cell death caused by sustained activation of the Hog1 stress-activated protein kinase. EMBO Rep. 2011, 2, 1062-1068. [CrossRef] [PubMed]

14. Day, A.M.; Smith, D.A.; Ikeh, M.A.C.; Haider, M.; Herrer -de-Dios, C.M.; Brown, A.J.P.; Morgan, B.A.; Erwig, L.P.; MacCallum, D.M.; Quinn, J. Blocking two-component signalling enhances Candida albicans virulence and reveals adaptive mechanisms that counteract sustained SAPK activation. PLoS Pathog. 2017, 13, e1006131. [CrossRef] [PubMed]

15. Bohnert, S.; Heck, L.; Gruber, C.; Neumann, H.; Distler, U.; Tenzer, S.; Yemelin, A.; Thines, E.; Jacob, S. Fungicide resistance toward fludioxonil conferred by overexpression of the phosphatase gene MoPTP2 in Magnaporthe oryzae. Mol. Microbiol. 2019, $111,662-677$.

16. Simaan, H.; Shalaby, S.; Hatoel, M.; Karinski, O.; Goldshmidt-Tran, O.; Horwitz, B.A. The AP-1-like transcription factor ChAP1 balances tolerance and cell death in the response of the maize pathogen Cochliobolus heterostrophus to a plant phenolic. Curr. Genet. 2020, 66, 187-203. [CrossRef]

17. Ohm, R.A.; Feau, N.; Henrissat, B.; Schoch, C.L.; Horwitz, B.A.; Barry, K.W.; Condon, B.J.; Copeland, A.C.; Dhillon, B.; Glaser, F.; et al. Diverse Lifestyles and Strategies of Plant Pathogenesis Encoded in the Genomes of Eighteen Dothideomycetes Fungi. PLoS Pathog. 2012, 8, e1003037. [CrossRef]

18. Ghosh, A.; Servin, J.A.; Park, G.; Borkovich, K.A. Global analysis of serine/threonine and tyrosine protein phosphatase catalytic subunit genes in Neurospora crassa reveals interplay between phosphatases and the p38 mitogen-activated protein kinase. G3 Genes Genomes Genet. 2014, 4, 349-365.

19. Winkelströter, L.K.; Dolan, S.K.; dos Reis, T.F.; Bom, V.L.P.; de Castro, P.A.; Hagiwara, D.; Alowni, R.; Jones, G.W.; Doyle, S.; Brown, N.A.; et al. Systematic global analysis of genes encoding protein phosphatases in Aspergillus fumigatus. G3 Genes Genomes Genet. 2015, 5, 1525-1539.

20. Jacoby, T.; Flanagan, H.; Faykin, A.; Seto, A.G.; Mattison, C.; Ota, I. Two protein-tyrosine phosphatases inactivate the osmotic stress response pathway in yeast by targeting the mitogen-activated protein kinase, Hog1. J. Biol. Chem. 1997, 272, 17749-17755. [CrossRef]

21. Ariño, J.; Casamayor, A.; González, A. Type 2C protein phosphatases in fungi. Eukaryot. Cell 2011, 10, 21-33. [CrossRef] [PubMed]

22. Gao, P.; Jin, K.; Xia, Y. The phosphatase gene MaCdc14 negatively regulates UV-B tolerance by mediating the transcription of melanin synthesis-related genes and contributes to conidiation in Metarhizium acridum. Curr. Genet. 2020, 66, 141-153. [CrossRef] [PubMed]

23. Rodriguez-Iglesias, A.; Schmoll, M. Protein Phosphatases Regulate Growth, Development, Cellulases and Secondary Metabolism in Trichoderma Reesei. Sci. Rep. 2019, 9. [CrossRef] [PubMed]

24. Wang, J.; Liu, J.; Hu, Y.; Ying, S.H.; Feng, M.G. Cytokinesis-required Cdc14 is a signaling hub of asexual development and multi-stress tolerance in Beauveria bassiana. Sci. Rep. 2013, 3, 1-8. [CrossRef] [PubMed]

25. Radmaneshfar, E.; Kaloriti, D.; Gustin, M.C.; Gow, N.A.R.; Brown, A.J.P.; Grebogi, C.; Romano, M.C.; Thiel, M. From START to FINISH: The Influence of Osmotic Stress on the Cell Cycle. PLoS ONE 2013, 8, e68067. [CrossRef]

26. Kaneva, I.N.; Sudbery, I.M.; Dickman, M.J.; Sudbery, P.E. Proteins that physically interact with the phosphatase Cdc14 in Candida albicans have diverse roles in the cell cycle. Sci. Rep. 2019, 9, 6258. [CrossRef] 
27. Lee, K.T.; Byun, H.J.; Jung, K.W.; Hong, J.; Cheong, E.; Bahn, Y.S. Distinct and redundant roles of protein tyrosine phosphatases Ptp1 and Ptp2 in governing the differentiation and pathogenicity of Cryptococcus neoformans. Eukaryot. Cell 2014, 13, 796-812. [CrossRef]

28. Dereeper, A.; Guignon, V.; Blanc, G.; Audic, S.; Buffet, S.; Chevenet, F.; Dufayard, J.F.; Guindon, S.; Lefort, V.; Lescot, M.; et al. Phylogeny.fr: Robust phylogenetic analysis for the non-specialist. Nucleic Acids Res. 2008, 36, W465-W469. [CrossRef]

29. Catlett, N.L.; Lee, B.-N.; Yoder, O.C.; Turgeon, B.G. Split-Marker Recombination for Efficient Targeted Deletion of Fungal Genes. Fungal Genet. Rep. 2003, 50, 9-11. [CrossRef]

30. Turgeon, B.G.; Condon, B.; Liu, J.; Zhang, N. Protoplast transformation of filamentous fungi. Methods Mol. Biol. $2010,638,3-19$.

31. Gurtovenko, A.A.; Anwar, J. Modulating the Structure and Properties of Cell Membranes: The Molecular Mechanism of Action of Dimethyl Sulfoxide. J. Phys. Chem. B 2007. [CrossRef] [PubMed]

32. Kojima, K.; Takano, Y.; Yoshimi, A.; Tanaka, C.; Kikuchi, T.; Okuno, T. Fungicide Activity through Activation of a Fungal Signalling Pathway. Mol. Microbiol. 2004. [CrossRef] [PubMed]

33. Pérez-Nadales, E.; di Pietro, A. The Membrane Mucin Msb2 Regulates Invasive Growth and Plant Infection in Fusarium oxysporum. Plant Cell 2011, 23, 1171-1185. [CrossRef] [PubMed]

34. Jandric, Z.; Gregori, C.; Klopf, E.; Radolf, M.; Schüller, C. Sorbic Acid Stress Activates the Candida glabrata High Osmolarity Glycerol MAP Kinase Pathway. Front. Microbiol. 2013. [CrossRef]

35. Igbaria, A.; Lev, S.; Rose, M.S.; Bee, N.L.; Hadar, R.; Degani, O.; Horwitz, B.A. Distinct and combined roles of the MAP kinases of Cochliobolus heterostrophus in virulence and stress responses. Mol. Plant-Microbe Interact. 2008, 21, 769-780. [CrossRef]

36. Bahn, Y.S.; Kojima, K.; Cox, G.M.; Heitman, J. Specialization of the HOG pathway and its impact on differentiation and virulence of Cryptococcus neoformans. Mol. Biol. Cell 2005, 16, 2285-2300. [CrossRef]

37. Pearson, G.; Robinson, F.; Beers Gibson, T.; Xu, B.; Karandikar, M.; Berman, K.; Cobb, M.H. Mitogen-Activated Protein (MAP) Kinase Pathways: Regulation and Physiological Functions. Endocr. Rev. 2001, 22, 153-183.

38. Stegmeier, F.; Amon, A. Closing mitosis: The functions of the Cdc14 phosphatase and its regulation. Annu. Rev. Genet. 2004, 38, 203-232. [CrossRef]

39. Zhan, X.L.; Hong, Y.; Zhu, T.; Mitchell, A.P.; Deschenes, R.J.; Guan, K.L. Essential functions of protein tyrosine phosphatases Ptp2 and Ptp3 and Rim11 tyrosine phosphorylation in Saccharomyces cerevisiae meiosis and sporulation. Mol. Biol. Cell 2000, 11, 663-676. [CrossRef] 